\title{
Rare fluctuations of the $S$ matrix at NLO in QCD
}

\author{
Wenchang Xiang, ${ }^{1,2 *}$ Yanbing Cai, ${ }^{1, \dagger}$ Mengliang Wang, ${ }^{1, *}$ and Daicui Zhou ${ }^{3, \S}$ \\ ${ }^{1}$ Guizhou Key Laboratory in Physics and Related Areas, Guizhou University of Finance and Economics, \\ Guiyang 550025, China \\ ${ }^{2}$ Department of Physics, Guizhou University, Guiyang 550025, China \\ ${ }^{3}$ Key Laboratory of Quark and Lepton Physics (MOE) and Institute of Particle Physics, Central China \\ Normal University, Wuhan 430079, China
}

(Received 6 January 2019; published 30 May 2019)

\begin{abstract}
We calculate the rare fluctuations of the $S$ matrix for high-energy dipole-dipole scattering on top of the full next-to-leading-order corrections in the center of mass frame. The relevant result in the saturation regime shows that the exponential factor of the $S$ matrix is $\sqrt{2}$ as large as the result which emerges when the rare fluctuation effects are taken into account. We find that the factor of $\sqrt{2}$ change of the exponential factor is induced by the gluon loop corrections which compensate for part of the rapidity decrease of the $S$ matrix made by quark loops and lead to the rare fluctuations becoming important again. To ensure the relevant results of the $S$ matrix are independent of the frame choice, the rare fluctuations of the $S$ matrix are also derived in a general frame. It is found that all the results are consistent with each other in both frames.
\end{abstract}

DOI: 10.1103/PhysRevD.99.096026

\section{INTRODUCTION}

In high-energy QCD, one of the most challenging problem has been to study theoretically and experimentally the parton saturation. This saturation phenomenon was first introduced by Gribov, Levin, and Ryskin in 1983 and was developed as a dual description of unitarity [1]. Since then, many physicists are engaged in searching of QCD evolution equations for describing the evolution of high-energy and density gluon systems. Among them, the most widely used one is the Balitsky-Kovchegov (BK) equation [2,3] because of its relatively simple structure. The BK equation includes the nonlinear effect, which ensures the scattering amplitude fully satisfying the unitarity constraints instead of an exponential growth with rapidity in the BalitskyFadin-Kuraev-Lipatov (BFKL) dynamics [4,5]. At leading logarithmic accuracy where the BK equation resums large logarithms $\alpha_{s} \ln (1 / x)$ corrections with fixed coupling constant $\alpha_{s}$, the analytical [6,7] and numerical [8] studies of the BK equation show that the BK equation with only a leading-order (LO) contribution cannot precisely describe the high-energy scattering in the small- $x$ region.

\footnotetext{
*wxiangphy@gmail.com

†myparticle@163.com

*mengliang.wang@mail.gufe.edu.cn

\$dczhou@mail.ccnu.edu.cn
}

Published by the American Physical Society under the terms of the Creative Commons Attribution 4.0 International license. Further distribution of this work must maintain attribution to the author(s) and the published article's title, journal citation, and DOI. Funded by SCOAP ${ }^{3}$.
In the past decade, the remarkable developments beyond leading logarithmic accuracy have been made to systematically improve the precision of the BK equation. One of the most important efforts was the consideration of the running coupling corrections. The running coupling BK (rcBK) equations were independently derived by Balitsky in Ref. [9] and Kovchegov and Weigert in Ref. [10] via resummation all order corrections associated with the coupling. However, the rcBK equations resulting from these two groups have different formats due to using different separation schemes between the running coupling and subtraction. Fortunately, in the saturation region (the most interesting regime in this paper), our studies in Ref. [11] show that these two rcBK equations reduce to a uniform format and have a same solution. In a full region, the numerical solutions to the rcBK equations show that the evolution speed of the scattering amplitude from Balitsky's derivation is dramatically suppressed compared to the one from the fixed coupling BK equation [12], which coincides with the theoretical expectations. Although the running coupling corrections significantly slow down the evolution speed of the dipole amplitude as rapidity increasing, they are not the only higher-order corrections relative to the LO BK equation. Indeed in the language of Feynman diagram, the running coupling corrections are only the contribution from quark loops, as we know that the gluon loops also have a large contribution to the evolution kernel [13]. By combining the contributions from quark and gluon loops, Balitsky and Chirilli got a full next-to-leading-order (NLO) BK equation [13]. The first numerical solution of the full NLO BK equation shows that the solution is sensitive to 
the details of the initial condition and becomes negative and nonvanishing at very small dipole size, which is unphysical [14]. The origin of the instability of the solution is due to the evolution equation including a large double transverse logarithmic correction term $\left(\sim \ln \left[\left(x_{\perp}-z_{\perp}\right)^{2} /\right.\right.$ $\left.\left.\left(x_{\perp}-y_{\perp}\right)^{2}\right] \ln \left[\left(y_{\perp}-z_{\perp}\right)^{2} /\left(x_{\perp}-y_{\perp}\right)^{2}\right]\right)$. Fortunately, the studies in Ref. [15] found that the instability problem can be solved by a resummation scheme for the double transverse logarithms.

Parallel to the developments in the calculations of contributions from quark and gluon loops, another effort in higher-order contributions is also performed by including the effect of rare fluctuations to improve the precision of the BK equation [11,16-20]. As discussed by Iancu and Mueller in Ref. [20], a typical configuration includes too many gluons at the time of collision, therefore leading to a very small $S$ matrix. A rare configuration containing a small number of gluons in the wave function has been found by suppressing the evolution of the gluons, which can lead to a relatively large $S$ matrix. In the fixed coupling case, the $S$ matrix obtained from the BK equation shows a quadratic rapidity dependence in its exponent, and it was found that the exponential factor of the $S$ matrix $\left(\sim \exp \left[-c_{1} \bar{\alpha}_{S}^{2}\left(Y-Y_{0}\right)^{2} / 2\right]\right)$ is twice as large as the one which takes into account the rare fluctuation effects [20], where $\bar{\alpha}_{s}$ is the coupling constant, and $c_{1}$ is a constant which is not important in this paper. This result shows that the rare fluctuations reduce the evolution speed of the dipole scattering amplitude with respect to rapidity. We would like to note that the result is obtained by including the rare fluctuations on top of the fixed coupling. How about the result for the rare fluctuations on top of the running coupling?

From the above discussions, we know that both running coupling and rare fluctuation effects can lead to suppress the evolution speed of the dipole scattering amplitude. To reveal which one is the dominant effect, we studied the rare fluctuations on top of the running coupling effect in Ref. [11]. First, we solved the rcBK equation in the saturation region to get an analytic $S$ matrix $\left(\sim \exp \left[-N_{c} \mu\left(Y-Y_{0}\right) / \pi \mu_{1}\right]\right.$ with $\mu$ and $\mu_{1}$ coming from running coupling at one-loop accuracy) which shows a linear rapidity dependence in its exponent. Then the rare fluctuations is computed on top of this $S$ matrix, it was found that the rare fluctuation effects take a negligible change of the exponent of the $S$ matrix, which mean that the rare fluctuations are less important in the running coupling case than in the fixed coupling case.

Recently, the solution to the full NLO BK equation was derived in the saturation region [21]. The analytic result of the $S$ matrix shows that the $\exp (-\mathcal{O}(Y))$ rapidity dependence of the running coupling solution is replaced by $\exp \left(-\mathcal{O}\left(Y^{3 / 2}\right)\right)$ in the NLO solution. As we know that the rare fluctuations in the fixed coupling case shows a significant suppression of the dipole amplitude, we believe that the rare fluctuations in the full NLO case would also play an important role in the evolution of dipole amplitude, although the rare fluctuations are less important in the running coupling case.

In this paper, we investigate the rare fluctuations of the $S$ matrix for high-energy dipole-dipole scattering on top of full NLO corrections in the saturation region. To see the rare fluctuation effects, we first recall the analytic solutions of LO, running coupling, and NLO BK equations in the saturation region, and we then study the rare fluctuation effects on top of these solutions. We find an interesting result, that the exponential factor of the $S$ matrix from the NLO BK equation without rare fluctuations is about $\sqrt{2}$ times larger than the one with rare fluctuations. The result shows that the influence of the rare fluctuations in the NLO BK case on the $S$ matrix are greater than that in the running coupling BK case, which indicates that the rare fluctuations are important in the NLO BK case, although it is not as significant as the LO BK case.

\section{LEADING-ORDER EVOLUTION EQUATION}

As we know, the simplest way to describe the scattering of a quark-antiquark dipole on a target (maybe another dipole, hadron, or nucleus) in the high-energy regime is the BK equation, which is a mean field version of the Balitsky-JIMWLK ${ }^{1}$ hierarchy [22-25] equations. The BK equation resums leading logarithmic $\alpha_{s} \ln (1 / x)$ corrections with fixed coupling constant $\alpha_{s}$, which is a leading-order equation.

\section{A. Balitsky-Kovchegov equation}

Consider a scattering of a quark-antiquark dipole with a quark at transverse coordinate $x_{\perp}$ and an antiquark at transverse coordinate $y_{\perp}$ on a target, where the dipole is left moving (unevolved), and the target is right moving (highly evolved); we usually call this frame a dipole frame [26]. In this frame, almost all of the relative rapidity between the dipole and the target, $Y$, is taken by the target. If one increases the rapidity of the dipole by a small amount $d Y$ while keeping the rapidity of the target fixed, then the dipole has a probability of emitting a gluon at transverse coordinate $z_{\perp}$ due to the rapidity change. In the large $N_{c}$, limit the quark-antiquark-gluon state can be viewed as a system of two dipoles, which means a parent dipole splitting into two daughter dipoles. In the fixed coupling case, this evolution can be described by the following equation [26],

${ }^{1}$ JIMWLK is the abbreviation for Jalilian-Marian, Iancu, McLerran, Weigert, Leonidov, and Kovner. 


$$
\frac{\partial}{\partial Y} S(r, Y)=\int d^{2} r_{1} K^{\mathrm{LO}}\left(r, r_{1}, r_{2}\right)\left[S^{(2)}\left(r_{1}, r_{2}, Y\right)-S(r, Y)\right],
$$

where the evolution kernel is given by

$$
K^{\mathrm{LO}}\left(r, r_{1}, r_{2}\right)=\frac{\bar{\alpha}_{s}}{2 \pi} \frac{r^{2}}{r_{1}^{2} r_{2}^{2}},
$$

with $\bar{\alpha}_{s}=\alpha_{s} N_{c} / \pi$. Here we use the notation $\mathbf{r}=x_{\perp}-y_{\perp}$ as the transverse size of parent dipole and $\mathbf{r}_{1}=x_{\perp}-z_{\perp}$ and $\mathbf{r}_{2}=z_{\perp}-y_{\perp}$ as the transverse sizes of the two emitted daughter dipoles, respectively. It is easy to see that Eq. (1) has a nonlinear term on the right-hand side, which accounts for the simultaneous scattering of the two daughter dipoles on the target. Equation (1) is almost impossible to use directly since a solution for $S(r, Y)$ desires knowing $S^{(2)}\left(r_{1}, r_{2}, Y\right)$. In the mean field approximation, one can simplify the nonlinear term as follows:

$$
S^{(2)}\left(r_{1}, r_{2}, Y\right) \simeq S\left(r_{1}, Y\right) S\left(r_{2}, Y\right) .
$$

Substituting Eq. (3) into Eq. (1), one obtains the BK equation

$$
\begin{aligned}
& \frac{\partial}{\partial Y} S(r, Y) \\
& \quad=\int d^{2} r_{1} K^{\mathrm{LO}}\left(r, r_{1}, r_{2}\right)\left[S\left(r_{1}, Y\right) S\left(r_{2}, Y\right)-S(r, Y)\right],
\end{aligned}
$$

which is a closed equation and can be solved analytically in the saturation region. With $N(r, Y)=1-S(r, Y)$, another version of the $\mathrm{BK}$ equation is obtained

$$
\begin{aligned}
\frac{\partial}{\partial Y} N(r, Y)= & \int d^{2} r_{1} K^{\mathrm{LO}}\left(r, r_{1}, r_{2}\right)\left[N\left(r_{1}, Y\right)\right. \\
& \left.+N\left(r_{2}, Y\right)-N(r, Y)-N\left(r_{1}, Y\right) N\left(r_{2}, Y\right)\right] .
\end{aligned}
$$

On the right-hand side of Eq. (5), the first and second terms describe the scattering of a single dipole with target, and the third term is a virtual term which expresses the probability for the parent dipole not to evolve, i.e., not to radiate small $x$ gluons. The virtual term can also be interpreted as the parent dipole survival probability. The nonlinear term takes into account a simultaneous interaction of two produced dipoles with the target.

\section{B. Analytic solution in the saturation region}

In the saturation region where the unitarity corrections become important or $S$ is very small, the nonlinear term in Eq. (4) is much smaller than the linear term. Therefore, the nonlinear term can be neglected, and Eq. (4) simplifies to

$$
\frac{\partial}{\partial Y} S(r, Y)=-\int d^{2} r_{1} K^{\mathrm{LO}}\left(r, r_{1}, r_{2}\right) S(r, Y) .
$$

Since the dipole size is much larger than the characteristic size $1 / Q_{s}$ in the saturation regime, the lower bound of integration in Eq. (6) can be set to $1 / Q_{s}$, where $Q_{s}$ is the saturation scale. We set the upper bound of the integration to $r$ due to a rapid decrease of the integration beyond $r$. By analyzing the kernel of the integration in Eq. (6), one can know that the integration is dominated by the region either from $1 / Q_{s} \ll\left|\mathbf{r}_{\mathbf{1}}\right| \ll|\mathbf{r}|,\left|\mathbf{r}_{\mathbf{2}}\right| \sim|\mathbf{r}|$ or from $1 / Q_{s} \ll\left|\mathbf{r}_{\mathbf{2}}\right| \ll$ $|\mathbf{r}|,\left|\mathbf{r}_{1}\right| \sim|\mathbf{r}|$. Suppose that we work in the region $\left|\mathbf{r}_{2}\right| \sim|\mathbf{r}|$, and that the kernel in Eq. (6) becomes $1 / r_{1}^{2}$, which significantly simplifies the calculations. Now it is easy to get the analytic solution of the BK equation by performing the integrations over $r_{1}$ and $Y$ [26,27],

$$
S(r, Y)=\exp \left[-\frac{c}{2} \bar{\alpha}_{S}^{2}\left(Y-Y_{0}\right)^{2}\right] S\left(r, Y_{0}\right),
$$

where we have used $Q_{s}^{2}(Y)=\exp \left[c \bar{\alpha}_{s}\left(Y-Y_{0}\right)\right] Q_{s}^{2}\left(Y_{0}\right)$ with $Q_{s}^{2}\left(Y_{0}\right) r^{2}=1$. From Eq. (7), we can see that the analytic solution of the LO BK equation has a quadratic rapidity dependence in the exponent. However, we will see in the following sections that the solution will be modified by taking into account the higher-order contributions, especially rare fluctuation effects. The reason why we have gone through such a detailed "derivation" of Eq. (7) is that the major aim of this study is to show how the $S$ matrix is modified by rare fluctuation effects in the cases of LO, running coupling and NLO cases, respectively.

\section{NEXT-TO-LEADING-ORDER EVOLUTION EQUATION}

The LO BK equation discussed above considers only the resummation of leading logarithmic $\alpha_{s} \ln \left(1 / x_{B j}\right)$ corrections with a fixed coupling constant. Beyond the leading logarithmic approximation, significant progress in the evolution equation has been made via the resummation of $\alpha_{s} N_{f}$ to all orders, which is usually called the running coupling corrections $[9,10]$.

\section{A. Running coupling Balitsky-Kovchegov equation and its analytic solution}

When one resums all powers of $\alpha_{s} N_{f}$ in the evolution kernel, the $\alpha_{s} N_{f}$ corrections modify the structure of the evolution equation. The evolution equation with running coupling corrections can be expressed as [12]

$$
\begin{aligned}
\frac{\partial S\left(x_{\perp}-y_{\perp}, Y\right)}{\partial Y} \\
=\int d^{2} z_{\perp} K^{r c}\left(x_{\perp}, y_{\perp}, z_{\perp}\right)\left[S\left(x_{\perp}-z_{\perp}, Y\right) S\left(z_{\perp}-y_{\perp}, Y\right)\right. \\
\left.\quad-S\left(x_{\perp}-y_{\perp}, Y\right)\right] \\
\quad-\alpha_{\mu}^{2} \int d^{2} z_{\perp 1} d^{2} z_{\perp 2} K_{\odot}\left(x_{\perp}, y_{\perp} ; z_{\perp 1}, z_{\perp 2}\right) \\
\quad \times\left[S\left(x_{\perp}-w_{\perp}, Y\right) S\left(w_{\perp}-y_{\perp}, Y\right)\right. \\
\left.\quad-S\left(x_{\perp}-z_{\perp 1}, Y\right) S\left(z_{\perp 2}-y_{\perp}, Y\right)\right],
\end{aligned}
$$


where $w_{\perp}$ is the subtraction point which can be chosen to be the transverse coordinate of the emitted gluon $z_{\perp}$ or the transverse coordinate of either the quark $z_{\perp 1}$ or the antiquark $z_{\perp 2}$. From Eq. (8), we know that it has two parts, the "running coupling" part [the first line on the rhs of Eq. (8)] and the "subtraction" part [the second line on the rhs of Eq. (8)]. The running coupling part has the same structure as the LO BK evolution equation but with a modified kernel $K^{r c}$. The subtraction part has a new structure with two quadratic terms and a resummed JIMWLK kernel $K_{\oplus}$. It has been found that the separation between the running coupling and subtraction contributions is not unique, which depends on the choice of subtraction point $[9,10]$. Fortunately, we are only interesting in the analytic solution of the evolution equation in the saturation region in which the running coupling $\mathrm{BK}$ equation is independent of the selection of subtraction point [11] since, in this regime, the $S$ matrix is so small that its quadratic terms in Eq. (8) can be neglected. Therefore, the running coupling BK evolution equation simplifies to

$$
\frac{\partial S(r, Y)}{\partial Y}=-\int d^{2} z_{\perp} K^{r c}\left(r, r_{1}, r_{2}\right) S(r, Y),
$$

where the modified kernel $K^{r c}\left(r, r_{1}, r_{2}\right)$ has two kinds of expressions (Balitsky and Kovchegov-Weigert kernels) since two different separation schemes have been used (please see Refs. [11,12] for more details about the kernels). Although Balitsky and Kovchegov-Weigert kernels have a different format at a glance, it has been shown in Ref. [11] that both kernels reduce to a unique form, Eq. (12), in the saturation region. In this study, we adopt the choice proposed by Kovchegov and Weigert in Ref. [10], and the modified kernel is written as [10]

$$
\begin{aligned}
& K^{r c K W}\left(r, r_{1}, r_{2}\right) \\
& \quad=\frac{N_{c}}{2 \pi^{2}}\left[\alpha_{s}\left(r_{1}^{2}\right) \frac{1}{r_{1}^{2}}-2 \frac{\alpha_{s}\left(r_{1}^{2}\right) \alpha_{s}\left(r_{2}^{2}\right)}{\alpha_{s}\left(R^{2}\right)} \frac{\mathbf{r}_{1} \cdot \mathbf{r}_{2}}{r_{1}^{2} r_{2}^{2}}+\alpha_{s}\left(r_{2}^{2}\right) \frac{1}{r_{2}^{2}}\right],
\end{aligned}
$$

with

$$
R^{2}\left(r, r_{1}, r_{2}\right)=r_{1} r_{2}\left(\frac{r_{2}}{r_{1}}\right)^{\frac{r_{1}^{2}+r_{2}^{2}}{r_{1}^{2}-r_{2}^{2}}-2 \frac{r_{1}^{2} r_{2}^{2}}{r_{1} \cdot r_{2} r_{1}^{2}-r_{2}^{2}}}
$$

In the saturation regime, the dominant integral region on the rhs of Eq. (9) comes from either $1 / Q_{s} \ll\left|\mathbf{r}_{1}\right| \ll|\mathbf{r}|,\left|\mathbf{r}_{\mathbf{2}}\right| \sim$ $|\mathbf{r}|$ or $1 / Q_{s} \ll\left|\mathbf{r}_{\mathbf{2}}\right| \ll|\mathbf{r}|,\left|\mathbf{r}_{1}\right| \sim|\mathbf{r}|$. In this work, we choose the first one, and the modified kernel becomes

$$
K^{r c K W}\left(\mathbf{r}, \mathbf{r}_{1}, \mathbf{r}_{2}\right)=\frac{N_{c}}{2 \pi^{2}} \alpha_{s}\left(r_{1}^{2}\right) \frac{1}{r_{1}^{2}} .
$$

We would like to note that if one works in the second region, the same result should be obtained. It is worth pointing out that Eq. (12) is the same as the kernel obtained in the case of $r \gg 1 / Q_{s}^{2}$ in Ref. [28], in which the authors aimed to show that the geometric scaling of the scattering amplitude is violated in the case of the running coupling.

Here $\alpha_{s}$ is not fixed, and we use the running coupling at one-loop accuracy,

$$
\alpha_{s}\left(r_{1}^{2}\right)=\frac{\mu}{1+\mu_{1} \ln \left(\frac{1}{r_{1}^{2} \Lambda^{2}}\right)} .
$$

Substituting the simplified kernel (12) into Eq. (9) and using Eq. (13), we can get the running coupling BK equation in the saturation region

$$
\frac{\partial S(r, Y)}{\partial Y}=-2 \frac{N_{c}}{2 \pi^{2}} \int_{1 / Q_{s}^{2}}^{r^{2}} d^{2} r_{1} \frac{\alpha_{s}\left(r_{1}^{2}\right)}{r_{1}^{2}} S(r, Y),
$$

whose analytic solution is [11]

$$
\begin{aligned}
S(r, Y)= & \exp \left[-\frac{N_{c} \mu}{c \pi \mu_{1}}\left(\ln ^{2}\left(\frac{Q_{s}^{2}(Y)}{\Lambda^{2}}\right) \ln \left(\frac{1+\mu_{1} \ln \frac{Q_{s}^{2}(Y)}{\Lambda^{2}}}{1+\mu_{1} \ln \frac{1}{r^{2} \Lambda^{2}}}-\frac{1}{2}\right)\right.\right. \\
& +\frac{1}{\mu_{1}} \ln \left(\frac{Q_{s}^{2}(Y)}{\Lambda^{2}}\right) \\
& \left.\left.-\frac{1}{\mu_{1}^{2}} \ln \left(1+\mu_{1} \ln \frac{Q_{s}^{2}(Y)}{\Lambda^{2}}\right)\right)\right] S\left(r, Y_{0}\right)
\end{aligned}
$$

with $[1,6,29]$

$$
\ln \left(Q_{s}^{2}(Y) / \Lambda^{2}\right)=\sqrt{c\left(Y-Y_{0}\right)}+\mathcal{O}\left(Y^{1 / 6}\right) .
$$

Interestingly, Eq. (15) is consistent with the running coupling solution which is obtained by naive extending of the LO BK equation to the running coupling case in Ref. [30]. From the first term in the exponent in Eq. (15), one can see that the $S$ matrix has an independence on the dipole size $r$, which supports the intuitive guess of the violation of the geometric scaling behavior of the running coupling scattering amplitude in Ref. [28].

It is important to stress that the rapidity dependence of the $S$ matrix in the running coupling case is different from the one in the fixed coupling case. The exponent of the $S$ matrix in the running coupling case, Eq. (15), decreases linearly with rapidity, while the exponent of the $S$ matrix has a quadratic decrease with rapidity in the fixed coupling case; see Eq. (7).

\section{B. Full next-to-leading-order Balitsky-Kovchegov equation and its analytic solution}

In the above section, we discussed the running coupling modified BK equation, which considers only the 
contributions from quark loops. However, except for the quark loop contributions there are gluon loop contributions to the kernel of the evolution equation. A comprehensive corrections should include both the contributions from the quark and gluon loops, as well as from the tree gluon diagrams with quadratic and cubic nonlinearities [13]. Combining all of these contributions, one can get a full NLO evolution equation [13]

$$
\begin{aligned}
& \frac{\partial S\left(x_{\perp}-y_{\perp}, Y\right)}{\partial Y}=\frac{\bar{\alpha}_{s}}{2 \pi} \int \mathrm{d}^{2} z_{\perp} \frac{\left(x_{\perp}-y_{\perp}\right)^{2}}{\left(x_{\perp}-z_{\perp}\right)^{2}\left(y_{\perp}-z_{\perp}\right)^{2}}\left\{1+\frac{\bar{\alpha}_{s}}{4}\left[b \ln \left(x_{\perp}-y_{\perp}\right)^{2} \mu^{2}-b \frac{\left(x_{\perp}-z_{\perp}\right)^{2}-\left(y_{\perp}-z_{\perp}\right)^{2}}{\left(x_{\perp}-y_{\perp}\right)^{2}}\right.\right. \\
& \left.\left.\times \ln \frac{\left(x_{\perp}-z_{\perp}\right)^{2}}{\left(y_{\perp}-z_{\perp}\right)^{2}}+\frac{67}{9}-\frac{\pi^{2}}{3}-\frac{10 N_{\mathrm{f}}}{9 N_{\mathrm{c}}}-2 \ln \frac{\left(x_{\perp}-z_{\perp}\right)^{2}}{\left(x_{\perp}-y_{\perp}\right)^{2}} \ln \frac{\left(y_{\perp}-z_{\perp}\right)^{2}}{\left(x_{\perp}-y_{\perp}\right)^{2}}\right]\right\} \\
& \times\left[S\left(x_{\perp}-z_{\perp}, Y\right) S\left(z_{\perp}-y_{\perp}, Y\right)-S\left(x_{\perp}-y_{\perp}, Y\right)\right]+\frac{\bar{\alpha}_{s}^{2}}{8 \pi^{2}} \int \frac{\mathrm{d}^{2} z_{\perp} \mathrm{d}^{2} z_{\perp}^{\prime}}{\left(z_{\perp}^{\prime}-z_{\perp}\right)^{4}} \\
& \times\left\{\left[\frac{\left(x_{\perp}-z_{\perp}\right)^{2}\left(y_{\perp}-z_{\perp}^{\prime}\right)^{2}+\left(x_{\perp}-z_{\perp}^{\prime}\right)^{2}\left(y_{\perp}-z_{\perp}\right)^{2}-4\left(x_{\perp}-y_{\perp}\right)^{2}\left(z_{\perp}^{\prime}-z_{\perp}\right)^{2}}{\left(x_{\perp}-z_{\perp}\right)^{2}\left(y_{\perp}-z_{\perp}^{\prime}\right)^{2}-\left(x_{\perp}-z_{\perp}^{\prime}\right)^{2}\left(y_{\perp}-z_{\perp}\right)^{2}}+\frac{\left(x_{\perp}-y_{\perp}\right)^{2}\left(z_{\perp}^{\prime}-z_{\perp}\right)^{2}}{\left(x_{\perp}-z_{\perp}\right)^{2}\left(y_{\perp}-z_{\perp}^{\prime}\right)^{2}}\right.\right. \\
& \left.\left.+\frac{\left(x_{\perp}-y_{\perp}\right)^{4}\left(z_{\perp}^{\prime}-z_{\perp}\right)^{4}}{\left(x_{\perp}-z_{\perp}\right)^{2}\left(y_{\perp}-z_{\perp}^{\prime}\right)^{2}\left(\left(x_{\perp}-z_{\perp}\right)^{2}\left(y_{\perp}-z_{\perp}^{\prime}\right)^{2}-\left(x_{\perp}-z_{\perp}^{\prime}\right)^{2}\left(y_{\perp}-z_{\perp}\right)^{2}\right)}\right] \ln \frac{\left(x_{\perp}-z_{\perp}\right)^{2}\left(y_{\perp}-z_{\perp}^{\prime}\right)^{2}}{\left(x_{\perp}-z_{\perp}^{\prime}\right)^{2}\left(y_{\perp}-z_{\perp}\right)^{2}}-2\right\} \\
& \times\left[S\left(x_{\perp}-z_{\perp}^{\prime}, Y\right) S\left(z_{\perp}^{\prime}-z_{\perp}, Y\right) S\left(z_{\perp}-y_{\perp}, Y\right)-S\left(x_{\perp}-z_{\perp}^{\prime}, Y\right) S\left(z_{\perp}^{\prime}-y_{\perp}, Y\right)\right] \\
& +\frac{\bar{\alpha}_{s}^{2} N_{f}}{8 \pi^{2} N_{c}} \int \frac{\mathrm{d}^{2} z_{\perp}^{\prime} \mathrm{d}^{2} z_{\perp}}{\left(z_{\perp}^{\prime}-z_{\perp}\right)^{4}}\left[-\frac{\left(x_{\perp}-z_{\perp}^{\prime}\right)^{2}\left(y_{\perp}-z_{\perp}\right)^{2}+\left(x_{\perp}-z_{\perp}\right)^{2}\left(y_{\perp}-z_{\perp}^{\prime}\right)^{2}-\left(x_{\perp}-y_{\perp}\right)^{2}\left(z_{\perp}^{\prime}-z_{\perp}\right)^{2}}{\left(x_{\perp}-z_{\perp}\right)^{2}\left(y_{\perp}-z_{\perp}^{\prime}\right)^{2}-\left(x_{\perp}-z_{\perp}^{\prime}\right)^{2}\left(y_{\perp}-z_{\perp}\right)^{2}}\right. \\
& \left.\times \ln \frac{\left(x_{\perp}-z_{\perp}\right)^{2}\left(y_{\perp}-z_{\perp}^{\prime}\right)^{2}}{\left(x_{\perp}-z_{\perp}^{\prime}\right)^{2}\left(y_{\perp}-z_{\perp}\right)^{2}}+2\right]\left[S\left(x_{\perp}-z_{\perp}, Y\right) S\left(z_{\perp}^{\prime}-y_{\perp}, Y\right)-S\left(x_{\perp}-z_{\perp}^{\prime}, Y\right) S\left(z_{\perp}^{\prime}-y_{\perp}, Y\right)\right],
\end{aligned}
$$

where the $b=\left(11 N_{c}-2 N_{f}\right) / 3 N_{c}$ is the first coefficient of the $\beta$ function, $N_{f}$ is the number of flavors, and $\mu$ is the renormalization scale. The full NLO BK equation shows two remarkable features in its structure as compared to the LO BK equation. First, the single integration term which gets a correction of order $\mathcal{O}\left(\bar{\alpha}_{s}^{2}\right)$ to the evolution kernel has a similar structure to the LO BK equation. Second, there are two double integration terms, of order $\mathcal{O}\left(\bar{\alpha}_{s}^{2}\right)$, which contain only the nonlinear $S$ matrix. The double integrations over the transverse coordinates $z_{\perp}$ and $z_{\perp}^{\prime}$ refer to partonic fluctuations involving two additional partons (besides the original quark and the antiquark) at the time of collision. We use the notation $\mathbf{r}=x_{\perp}-y_{\perp}, \mathbf{r}_{1}=x_{\perp}-z_{\perp}, \mathbf{r}_{1}^{\prime}=x_{\perp}-z_{\perp}^{\prime}$, $\mathbf{r}_{2}=z_{\perp}-y_{\perp}$, and $\mathbf{r}_{2}^{\prime}=z_{\perp}{ }^{\prime}-y_{\perp}$ for the sizes of parent dipole and of the new daughter dipoles produced by the evolutions.

In the saturation regime, the unitarity corrections become important or $S$ is very small. Therefore, the nonlinear terms can be neglected in Eq. (17). The full NLO BK equation in saturation regime becomes

$$
\frac{\partial S(r, Y)}{\partial Y}=-\int \mathrm{d}^{2} z_{\perp} K^{f \mathrm{NLO}}\left(r, r_{1}, r_{2}\right) S(r, Y)
$$

with the modified kernel

$$
K^{f \mathrm{NLO}}\left(r, r_{1}, r_{2}\right)=\frac{\bar{\alpha}_{s}\left(r^{2}\right)}{2 \pi}\left\{\frac{r^{2}}{r_{1}^{2} r_{2}^{2}}+\frac{1}{r_{1}^{2}}\left[\frac{\alpha_{s}\left(r_{1}^{2}\right)}{\alpha_{s}\left(r_{2}^{2}\right)}-1\right]+\frac{1}{r_{2}^{2}}\left[\frac{\alpha_{s}\left(r_{2}^{2}\right)}{\alpha_{s}\left(r_{1}^{2}\right)}-1\right]+\frac{\bar{\alpha}_{s}\left(r^{2}\right)}{4} \frac{r^{2}}{r_{1}^{2} r_{2}^{2}}\left[\frac{67}{9}-\frac{\pi^{2}}{3}-\frac{10 N_{f}}{9 N_{c}}-2 \ln \frac{r_{1}^{2}}{r^{2}} \ln \frac{r_{2}^{2}}{r^{2}}\right]\right\} .
$$

To analytically solve Eq. (18), one should work in either the $1 / Q_{s} \ll\left|\mathbf{r}_{\mathbf{1}}\right| \ll|\mathbf{r}|,\left|\mathbf{r}_{\mathbf{2}}\right| \sim|\mathbf{r}|$ or the $1 / Q_{s} \ll\left|\mathbf{r}_{\mathbf{2}}\right| \ll$ $|\mathbf{r}|,\left|\mathbf{r}_{1}\right| \sim|\mathbf{r}|$ region, as mentioned in the LO case. If one chooses the first regime, the NLO kernel can simplify as follows:

$$
\begin{aligned}
K^{f \mathrm{NLO}}\left(r, r_{1}, r_{2}\right) & =\frac{\bar{\alpha}_{s}\left(r^{2}\right)}{2 \pi}\left[\frac{1}{r_{1}^{2}} \frac{\alpha_{s}\left(r_{1}^{2}\right)}{\alpha_{s}\left(r^{2}\right)}+\frac{1}{r^{2}}\left(\frac{\alpha_{s}\left(r^{2}\right)}{\alpha_{s}\left(r_{1}^{2}\right)}-1\right)+\frac{\bar{\alpha}_{s}\left(r^{2}\right)}{4} \frac{1}{r_{1}^{2}}\left(\frac{67}{9}-\frac{\pi^{2}}{3}-\frac{10 N_{f}}{9 N_{c}}-2 \ln \frac{r_{1}^{2}}{r^{2}} \ln \frac{r_{2}^{2}}{r^{2}}\right)\right] \\
& \simeq \frac{\bar{\alpha}_{s}\left(r_{1}^{2}\right)}{2 \pi r_{1}^{2}}+\frac{\bar{\alpha}_{s}^{2}\left(r^{2}\right)}{8 \pi r_{1}^{2}}\left(\frac{67}{9}-\frac{\pi^{2}}{3}-\frac{10 N_{f}}{9 N_{c}}\right) .
\end{aligned}
$$

Substituting the simplified kernel into Eq. (18), the evolution equation becomes 


$$
\frac{\partial S(r, Y)}{\partial Y}=-2 \frac{1}{2 \pi} \int_{1 / Q_{s}^{2}}^{r^{2}} \mathrm{~d}^{2} r_{1}\left[\frac{\bar{\alpha}_{s}\left(r_{1}^{2}\right)}{r_{1}^{2}}+\frac{\bar{\alpha}_{s}^{2}\left(r^{2}\right)}{4} \frac{1}{r_{1}^{2}}\left(\frac{67}{9}-\frac{\pi^{2}}{3}-\frac{10 N_{f}}{9 N_{c}}\right)\right] S(r, Y)
$$

and has the following solution [21],

$$
\begin{aligned}
S(r, Y)= & \exp \left[-\frac{N_{c} \mu}{c \pi \mu_{1}}\left(\frac{2 C_{r}}{3} \ln ^{3} \frac{Q_{s}^{2}(Y)}{\Lambda^{2}}+\ln ^{2} \frac{Q_{s}^{2}(Y)}{\Lambda^{2}} \ln \frac{\left(r^{2} \Lambda^{2}\right)^{C_{r}}+\left(r^{2} \Lambda^{2}\right)^{C_{r}} \mu_{1} \ln \frac{Q_{s}^{2}(Y)}{\Lambda^{2}}}{1+\mu_{1} \ln \frac{1}{\left(r^{2} \Lambda^{2}\right)}}\right.\right. \\
& \left.\left.+\frac{1}{\mu_{1}} \ln \frac{Q_{s}^{2}(Y)}{\Lambda^{2}}-\frac{1}{\mu_{1}^{2}} \ln \left(1+\mu_{1} \ln \frac{Q_{s}^{2}(Y)}{\Lambda^{2}}\right)\right)\right] S\left(r, Y_{0}\right),
\end{aligned}
$$

with $\ln \left(Q_{s}^{2}(Y) / \Lambda^{2}\right)=\sqrt{c\left(Y-Y_{0}\right)}+\mathcal{O}\left(Y^{1 / 6}\right)$ and $C_{r}=$ $\alpha_{s}^{2}\left(r^{2}\right) N_{c} \mu_{1}\left(67 / 9-\pi^{2} / 3-10 N_{f} / 9 N_{c}\right) / 4 \pi \mu$. From this solution, we can see that the full NLO corrections (quark plus gluon loop contributions) bring a large change to the $S$ matrix. The linear rapidity dependence of the running coupling solution, Eq. (15), is now replaced by the rapidity raised to the power of $3 / 2$ in the full NLO case; see Eq. (22). By comparing the solutions in the LO, running coupling, and full NLO cases, one can find that the running coupling effect (only quark loops) makes the quadratic rapidity $\left(\sim Y^{2}\right)$ dependence of the exponent of the $S$ matrix changing to linear $(\sim Y)$ dependence, while the full NLO effects (quark plus gluon loops) let the linear $Y$ dependence returning to $Y^{3 / 2}$ dependence since the gluon loop contributions compensate for part of the decrease. To doublecheck the analytic solution given by Eq. (22), we also derive the solution of Eq. (17) by using the method developed in Ref. [31]; see the Appendix for details. We find that the two solutions are consistent with each other.

We would like to note that the numerical solution of the full NLO BK equation shows that it is unstable, the dipole amplitude decreases with growing energy and can switch to a negative value [14]. It has been found that the instability comes from a large double-logarithmic contribution [15]. To solve this problem, one needs to resum double transverse logarithms to all orders and gets a double-logarithmic approximation (DLA) evolution equation which can be extending to full next-to-leading logarithmic (NLL) accuracy via including the quark and gluon loop contributions. We usually call this stabilized high-order evolution equation a NLL BK equation. Our previous studies in Ref. [21] found that, in the saturation region, the NLL BK equation has the same analytic solution as the NLO BK equation since the DLA kernel is equal to 1 under the saturation condition. This is why we neglect the detail derivation of the NLL BK equation and its solution in this study.

\section{EFFECTS OF RARE FLUCTUATIONS IN HIGH-ENERGY DIPOLE-DIPOLE SCATTERING IN THE CENTER OF MASS FRAME}

In the high-energy region where $S$ is small, rare fluctuation effects can play an important role in the evolution of the amplitude of dipole-dipole scattering [20]. These fluctuations are rare and unimportant in a general inelastic collision, but they can dramatically affect the $S$ matrix in the high-energy limit. The significance of the rare fluctuations can be viewed by comparing the following two scattering processes. First, at very high energy, if one carries out dipole-dipole scattering, the typical configuration of a dipole's light cone wave function is a color glass condensate at the time of scattering. Using this typical configuration to compute the $S$ matrix of this interaction in the center of mass frame at relative rapidity $Y$ in the saturation domain, ${ }^{2}$ the $S$ matrix of this scattering is proportional to $\exp \left[-\operatorname{const} Q_{S}^{2}(Y / 2) r^{2} / \alpha_{s}^{2}\right][20,32]$, which can be obtained by using $\ln S^{2}(r) \simeq-c_{0} \alpha_{s}^{2} N^{2}(r, r, Y / 2)$, with $N \simeq c_{1} Q_{s}^{2}(Y / 2) r^{2} / \alpha_{s}^{2}$, as the number of gluons in each wave function, $c_{0}$ and $c_{1}$ as the constant, and $r_{0}$ as the size of the parent dipole. Second, if one performs a scattering of a dipole (unevolved) on a nucleus (highly evolved) and calculates the $S$ matrix of this interaction process which can be described by using the LO BK equation, one gets the $S$ matrix to be proportional to $\exp \left[-\right.$ const $\left.\ln ^{2}\left(Q_{s}^{2} r^{2}\right)\right]$. By comparing the $S$ matrices of the two scattering processes mentioned above, we can see that the $S$ matrix of the dipoledipole scattering with two typical configurations is much smaller than the one from the dipole-nucleus scattering with only one typical configuration. It has been found that the inconsistency comes from the use of typical configurations to calculate the elastic scattering $S$ matrix [20]. Actually, in the saturation region, the $S$ matrix is dominated by rare configurations which are dilute states with few gluons.

\section{A. Leading-order case}

The BK equation is obtained under a mean field approximation, which uses the product $S\left(r_{1}, Y\right) S\left(r_{2}, Y\right)$ replacing $S^{(2)}\left(r_{1}, r_{2}, Y\right)$. This replacement is true only in the absence of fluctuations. It has been found that the $S$ matrix derived from the BK equation is too small since the wave functions

\footnotetext{
${ }^{2} \mathrm{We}$ would like to point out that it is not known how to calculate the $S$ matrix of dense-dense scattering in the framework of a color glass condensate due to the unknown Lagrangian, although several authors have made efforts to extend dilute-dilute and dilute-dense scattering to dense-dense scattering approximately in the literature [32-35]. We concentrate on the study of dipole-dipole scattering in this paper.
} 


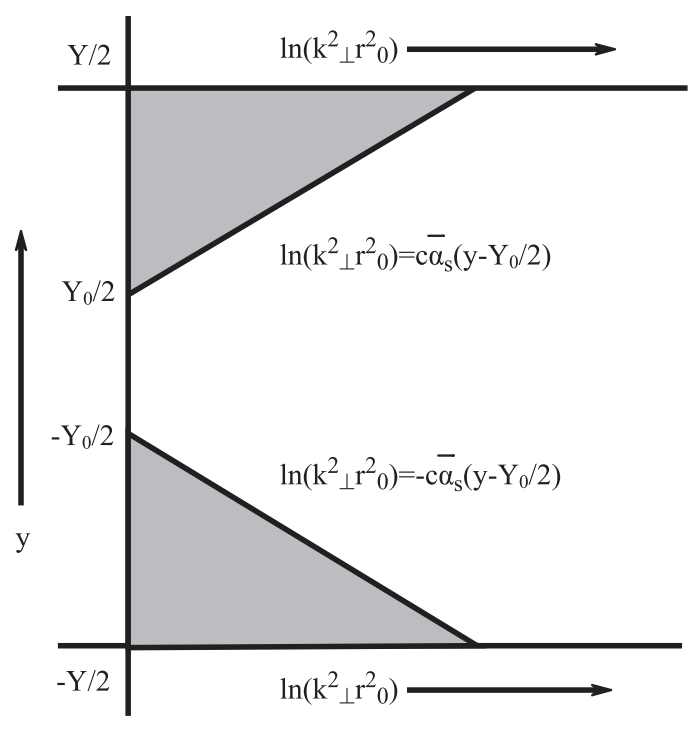

FIG. 1. The LO configuration in the center of mass frame.

contain too many gluons at the time of collision $[11,20]$. To get a relatively large $S$ matrix as compared to Eq. (7), one needs to search for a rare configuration which includes less than the mean number of gluons. The best way to find such a rare configuration is to minimize the number of gluons by suppressing the evolution of the system.

To suppress the evolution, we take a similar method which was used in Ref. [20]. Consider a central scattering of a right-moving dipole on a left-moving dipole in the center of mass frame at rapidity $Y>Y_{0}$, where $Y_{0}$ denotes the critical value for the onset of unitarity corrections. For the right-moving and left-moving dipoles, we require that the wave function of each dipole has no more than one dipole of size $r_{0}$ (being the size of the parent dipole) or larger in the rapidity intervals $Y_{0} / 2<y<Y / 2$ and $-Y / 2<y<Y_{0} / 2$, respectively.

To guarantee that this is the case, we need to suppress the emission of gluons (or creation of new dipoles ${ }^{3}$ ) from the parent dipole at $y>Y_{0} / 2$ in such a way that gluon emission is forbidden if gluon has $k_{\perp}$ and $y$ lying in the shaded triangles of Fig. 1, with $k_{\perp}$ being the momentum conjugate to $r_{\perp}$, because those emitted dipoles could evolve into dipoles of size $r_{0}$ or larger at rapidity $Y_{0} / 2$. However, we allow for creation of very small dipoles which locate on the right-hand side of the lines in Fig. 1. The line for the upper triangle in Fig. $1, \ln \left(k_{\perp}^{2} r_{0}^{2}\right)=c \bar{\alpha}_{s}\left(y-Y_{0} / 2\right)$ with $c \simeq 4.883$ [1], is determined by the requirement that the emitted dipoles located on the right-hand side of that line cannot evolve by normal BFKL evolution to give dipoles having $k_{\perp} \leq 1 / r_{0}$ at $Y_{0} / 2$. Turning to the rapidity interval $0<y<Y_{0} / 2$ just before the time of scattering at $y=0$, we let the wave function of the right-moving dipole undergoing a normal BFKL evolution.

\footnotetext{
${ }^{3}$ In the dipole model, a gluon can be equivalent to a $q \bar{q}$ dipole in the large $N_{c}$ limit.
}

We would like to note that a similar requirement is put into the evolution of the wave function of the left-moving dipole in $-Y / 2<y<Y_{0} / 2$ as for the aforementioned right-moving dipole in $Y_{0} / 2<y<Y / 2$. The wave function of the left-moving dipole also undergoes a normal BFKL evolution in $-Y_{0} / 2<y<0$ as the right-moving dipole in $0<y<Y_{0} / 2$.

By carrying out the operations mentioned above, the LO $S$ matrix including the rare fluctuations can be computed [20],

$$
S(r, Y)=A\left(r, \frac{Y-Y_{0}}{2}\right) A\left(r, \frac{Y-Y_{0}}{2}\right) S\left(r, Y_{0}\right),
$$

where $A\left(r,\left(Y-Y_{0}\right) / 2\right)$ is the probability of the rare configuration and has the same meaning as the parent dipole's survival probability after a BFKL evolution over a rapidity $Y-Y_{0}$ [20]. The terminology survival probability implies that $A\left(r, Y-Y_{0}\right)$ satisfies the same evolution equation as the virtual term in the LO BK equation (5),

$\frac{\partial}{\partial Y} A\left(r, Y-Y_{0}\right)=-\int d^{2} z_{1} K^{\mathrm{LO}}\left(\mathbf{r}, \mathbf{r}_{1}, \mathbf{r}_{2}\right) A\left(r, Y-Y_{0}\right)$.

Substituting the LO BK kernel into Eq. (24), one can get $\frac{\partial}{\partial Y} A\left(r, Y-Y_{0}\right)=-\int_{1 / Q_{s}^{2}}^{r^{2}} d^{2} r_{1} \frac{2 \bar{\alpha}_{s}}{2 \pi} \frac{r^{2}}{r_{1}^{2} r_{2}^{2}} A\left(r, Y-Y_{0}\right)$.

By a simple algebra calculation, we can obtain the solution of Eq. (25),

$$
\left.A\left(r, Y-Y_{0}\right)=\exp \left[-\frac{c}{2} \bar{\alpha}_{s}^{2}\left(Y-Y_{0}\right)^{2}\right)\right] .
$$

Taking this solution to Eq. (23), one can obtain [20]

$$
S(r, Y)=\exp \left[-\frac{c}{4} \bar{\alpha}_{S}^{2}\left(Y-Y_{0}\right)^{2}\right] S\left(r, Y_{0}\right),
$$

which is significantly larger than the one in Eq. (7) by comparing the exponent of the $S$ matrix. From Eq. (27), we can see that the exponential factor in Eq. (7) is twice as large as the result, Eq. (27), which includes the rare fluctuations. The rare fluctuations lead to the $S$ matrix becoming larger than the one coming from the LO BK equation, which indicates that the effects of rare fluctuations are very important in the LO case and cannot be neglected when one studies gluon saturation phenomenology in the saturation region at fixed coupling constant.

We would like to point out that one can use $S^{2}$ as the survival probability instead of $A(r, Y)$ since $S^{2}$ can be interpreted as the probability that no interaction occurs in the collision. However, we find that the $S$ matrix with rare fluctuations keeps the same form as the one without 
fluctuations once $S^{2}$ is used as the survival probability, which indicates that it seems the rare fluctuations make no contribution to the $S$ matrix. The reason why we get this undesired result would come from two approximations used during the derivation of the $S$ matrix. One is $S^{(2)}\left(r_{1}, r_{2}, Y\right) \simeq$ $S\left(r_{1}, Y\right) S\left(r_{2}, Y\right)$ [see Eq. (3)]; such a replacement is true only in the absence of fluctuations in the wave function of the target. Another approximation is that we assume the $S$ is small, and we neglect $S^{2}$ when we solve the BK equation; see Eq. (6). The $S$ matrix, Eq. (7), is obtained under the condition that $S^{2}$ is negligible. Therefore, using $S^{2}$ as the survival probability could be problematic under these approximations. Fortunately, in Ref. [36], Kozlov and Levin used the Reggeon-like diagram technique based on the BFKL Pomerons to confirm the results of Ref. [20] whose arguments are directly followed by our paper. Thus, using $A(r, Y)$ as the survival probability, which is proposed in Ref. [20], is feasible. In the following studies, we use $A(r, Y)$ as the survival probability.

\section{B. Next-to-leading-order case}

In this subsection, we investigate the rare fluctuations on top of the running coupling and the full NLO effects. To get the rare configuration, we need to follow the steps in our previous studies [11]. Considering a right-moving dipole scattering off a left-moving dipole, one needs to constrain the wave functions of the right-moving and left-moving dipoles in order to let the system consisting only of the parent dipole itself with size $r_{0}$ in the rapidity intervals $Y_{0} / 2<y<Y / 2$ and $-Y / 2<y<-Y_{0} / 2$, respectively. However, one cannot require that all evolutions of the right-moving and left-moving dipoles are absent in the rapidity intervals mentioned above. A feasible step that one can take is to allow that the evolution can only produce very small dipoles with size much smaller than $r_{0}$ to avoid these dipoles evolving into dipoles with similar size as $r_{0}$ in intermediate rapidities, which can guarantee that the final dipole system at rapidity $Y_{0} / 2$ has no more than one dipole of size $r_{0}$ or larger; see Fig. 2. Note that the lines for the upper and lower triangles in Fig. $2, \ln \left(k_{\perp}^{2} r_{0}^{2}\right)=$

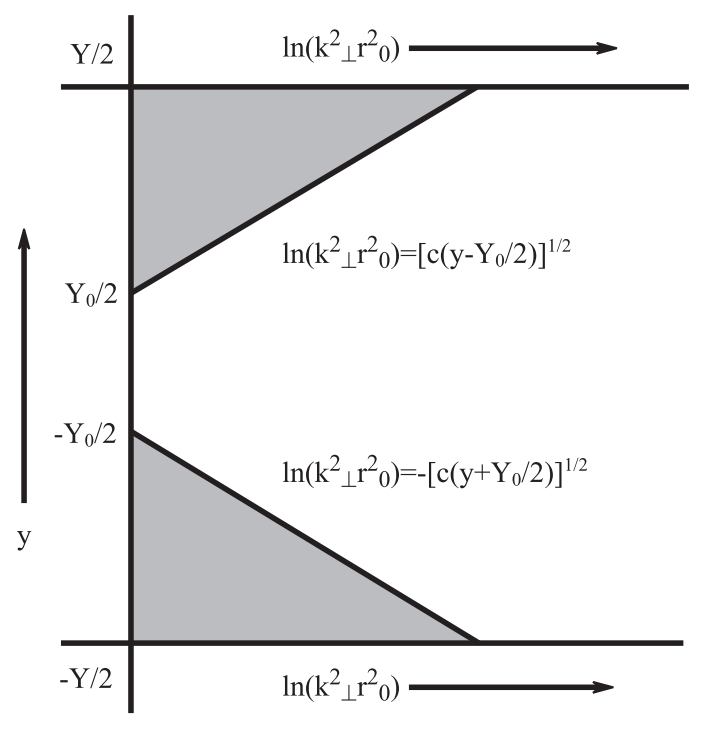

FIG. 2. The NLO configuration in the center of mass frame.

$\sqrt{c\left(y-Y_{0} / 2\right)}$ and $\ln \left(k_{\perp}^{2} r_{0}^{2}\right)=-\sqrt{c\left(y+Y_{0} / 2\right)}$, are determined as in the LO case except for the NLO corrections included here. So the linear rapidity dependence of the line in $\mathrm{LO}$ case is now replaced by square root dependence in the NLO case.

\section{In the case of running coupling}

We follow Eq. (23) to derive an $S$ matrix which includes both rare fluctuation and running coupling corrections. As in the LO case, the probability of a rare configuration in the running coupling case satisfies

$\frac{\partial}{\partial Y} A\left(r, Y-Y_{0}\right)=-\int d^{2} z_{1} K^{r c K W}\left(r, r_{1}, r_{2}\right) A\left(r, Y-Y_{0}\right)$,

where $K^{r c K W}\left(r, r_{1}, r_{2}\right)$ is the modified kernel which is given by Eq. (12). By solving the integro-differential equation, Eq. (28), one gets

$$
A\left(r, Y-Y_{0}\right)=\exp \left[-\frac{N_{c} \mu}{c \pi \mu_{1}}\left(\ln ^{2}\left(\frac{Q_{S}^{2}(Y)}{\Lambda^{2}}\right) \ln \left(\frac{1+\mu_{1} \ln \left(\frac{Q_{S}^{2}(Y)}{\Lambda^{2}}\right)}{1+\mu_{1} \ln \left(\frac{1}{r^{2} \Lambda^{2}}\right)}-\frac{1}{2}\right)+\frac{\ln \left(\frac{Q_{S}^{2}(Y)}{\Lambda^{2}}\right)}{\mu_{1}}-\frac{1}{\mu_{1}^{2}} \ln \left(1+\mu_{1} \ln \left(\frac{Q_{S}^{2}(Y)}{\Lambda^{2}}\right)\right)\right)\right] .
$$

By using Eq. (29), the $S$ matrix including both rare fluctuation and running coupling corrections can be computed as

$$
\begin{aligned}
S(r, Y) & =A\left(r, \frac{Y-Y_{0}}{2}\right) A\left(r, \frac{Y-Y_{0}}{2}\right) S\left(r, Y_{0}\right) \\
& =\exp \left[-\frac{N_{c} \mu}{c \pi \mu_{1}}\left(\ln ^{2}\left(\frac{Q_{S}^{2}(Y)}{\Lambda^{2}}\right) \ln \left(\frac{1+\frac{\mu_{1}}{\sqrt{2}} \ln \left(\frac{Q_{S}^{2}(Y)}{\Lambda^{2}}\right)}{1+\mu_{1} \ln \left(\frac{1}{r^{2} \Lambda^{2}}\right)}-\frac{1}{2}\right)+\frac{\sqrt{2} \ln \left(\frac{Q_{S}^{2}(Y)}{\Lambda^{2}}\right)}{\mu_{1}}-\frac{2}{\mu_{1}^{2}} \ln \left(1+\frac{\mu_{1}}{\sqrt{2}} \ln \left(\frac{Q_{S}^{2}(Y)}{\Lambda^{2}}\right)\right)\right)\right] S\left(r, Y_{0}\right),
\end{aligned}
$$


where $\ln \left(Q_{S}^{2}(Y) / \Lambda^{2}\right)=\sqrt{c\left(Y-Y_{0}\right)}$. To see whether or not the rare fluctuation effects bring a large change to the $S$ matrix, one can carry out a comparison between Eqs. (30) and (15). It is easy to find that, in the exponent of the $S$ matrix, the dominant terms are almost the same and the subterms have slight modifications, which means that the rare fluctuations are less important in the running coupling case than in the fixed coupling case, where the rare fluctuations correct the exponential factor of the $S$ matrix by a factor of 2 . The reason why the rare fluctuations are less important is that the running coupling makes the exponent of the $S$ matrix change to a linear rapidity dependence instead of a quadratic dependence in the fixed coupling case.

\section{In the case of full next-to-leading order}

The rare configuration in the full NLO case is similar to the one in the running coupling case; see Fig. 2. The probability of rare configuration $A\left(r, Y-Y_{0}\right)$ satisfies the evolution equation

$$
\begin{aligned}
& \frac{\partial}{\partial Y} A\left(r, Y-Y_{0}\right) \\
& \quad=-\int d^{2} z_{1} K^{f \mathrm{NLO}}\left(\mathbf{r}, \mathbf{r}_{1}, \mathbf{r}_{2}\right) A\left(r, Y-Y_{0}\right),
\end{aligned}
$$

whose solution is

$$
\begin{aligned}
A\left(r, Y-Y_{0}\right)= & \exp \left[-\frac{N_{c} \mu}{c \pi \mu_{1}}\left(\frac{2 C_{r}}{3}\left(c\left(Y-Y_{0}\right)\right)^{3 / 2}+c\left(Y-Y_{0}\right) \ln \frac{\left(r^{2} \Lambda^{2}\right)^{C_{r}}+\left(r^{2} \Lambda^{2}\right)^{C_{r}} \mu_{1}\left(c\left(Y-Y_{0}\right)\right)^{1 / 2}}{1+\mu_{1} \ln \frac{1}{\left(r^{2} \Lambda^{2}\right)}}\right.\right. \\
& \left.\left.+\frac{1}{\mu_{1}}\left(c\left(Y-Y_{0}\right)\right)^{1 / 2}-\frac{1}{\mu_{1}^{2}} \ln \left(1+\mu_{1}\left(c\left(Y-Y_{0}\right)\right)^{1 / 2}\right)\right)\right],
\end{aligned}
$$

where we have used $\ln \left(Q_{S}^{2}(Y) / \Lambda^{2}\right)=\sqrt{c\left(Y-Y_{0}\right)}$. To get the $S$ matrix in the center of mass frame, we need to compute

$$
\begin{aligned}
A\left(r, \frac{Y-Y_{0}}{2}\right)= & \exp \left[-\frac{N_{c} \mu}{c \pi \mu_{1}}\left(\frac{2 C_{r}}{3}\left(\frac{1}{2}\right)^{3 / 2}\left(c\left(Y-Y_{0}\right)\right)^{3 / 2}+\frac{c}{2}\left(Y-Y_{0}\right) \ln \frac{\left(r^{2} \Lambda^{2}\right)^{C_{r}}+\left(r^{2} \Lambda^{2}\right)^{C_{r}} \frac{\mu_{1}}{\sqrt{2}}\left(c\left(Y-Y_{0}\right)\right)^{1 / 2}}{1+\mu_{1} \ln \frac{1}{\left(r^{2} \Lambda^{2}\right)}}\right.\right. \\
& \left.\left.+\frac{1}{\sqrt{2} \mu_{1}}\left(c\left(Y-Y_{0}\right)\right)^{1 / 2}-\frac{1}{\mu_{1}^{2}} \ln \left(1+\frac{\mu_{1}}{\sqrt{2}}\left(c\left(Y-Y_{0}\right)\right)^{1 / 2}\right)\right)\right] .
\end{aligned}
$$

Then the $S$ matrix can be calculated as

$$
\begin{aligned}
& S(r, Y)=A\left(r, \frac{Y-Y_{0}}{2}\right) A\left(r, \frac{Y-Y_{0}}{2}\right) S\left(r, Y_{0}\right) \\
& =\exp \left[-\frac{N_{c} \mu}{c \pi \mu_{1}}\left(2\left(\frac{1}{2}\right)^{3 / 2} \frac{2 C_{r}}{3} \ln ^{3} \frac{Q_{s}^{2}(Y)}{\Lambda^{2}}+\ln ^{2} \frac{Q_{s}^{2}(Y)}{\Lambda^{2}} \ln \frac{\left(r^{2} \Lambda^{2}\right)^{C_{r}}+\left(r^{2} \Lambda^{2}\right)^{C_{r}} \frac{\mu_{1}}{\sqrt{2}} \ln \frac{Q_{s}^{2}(Y)}{\Lambda^{2}}}{1+\mu_{1} \ln \frac{1}{\left(r^{2} \Lambda^{2}\right)}}\right.\right. \\
& \left.\left.+\frac{\sqrt{2}}{\mu_{1}} \ln \frac{Q_{s}^{2}(Y)}{\Lambda^{2}}-\frac{2}{\mu_{1}^{2}} \ln \left(1+\frac{\mu_{1}}{\sqrt{2}} \ln \frac{Q_{s}^{2}(Y)}{\Lambda^{2}}\right)\right)\right] S\left(r, Y_{0}\right) \\
& =\exp \left[-\frac{N_{c} \mu}{c \pi \mu_{1}}\left(\frac{1}{\sqrt{2}} \frac{2 C_{r}}{3} \ln ^{3} \frac{Q_{s}^{2}(Y)}{\Lambda^{2}}+\ln ^{2} \frac{Q_{s}^{2}(Y)}{\Lambda^{2}} \ln \frac{\left(r^{2} \Lambda^{2}\right)^{C_{r}}+\left(r^{2} \Lambda^{2}\right)^{C_{r}} \frac{\mu_{1}}{\sqrt{2}} \ln \frac{Q_{s}^{2}(Y)}{\Lambda^{2}}}{1+\mu_{1} \ln \frac{1}{\left(r^{2} \Lambda^{2}\right)}}\right.\right. \\
& \left.\left.+\frac{\sqrt{2}}{\mu_{1}} \ln \frac{Q_{s}^{2}(Y)}{\Lambda^{2}}-\frac{2}{\mu_{1}^{2}} \ln \left(1+\frac{\mu_{1}}{\sqrt{2}} \ln \frac{Q_{s}^{2}(Y)}{\Lambda^{2}}\right)\right)\right] S\left(r, Y_{0}\right) .
\end{aligned}
$$

By comparing Eq. (34) with Eq. (22), we can clearly see that the exponential factor of the dominant term in Eq. (22) is $\sqrt{2}$ times larger than the one which presents when the rare fluctuation corrections are taken into account. This result shows that the rare fluctuation effects become important again in the full NLO case, which is not like the case of running coupling, where the rare fluctuations are washed away by the quark loop corrections. Before we explain the reason why the rare fluctuations become important again in the full NLO case, we do an analysis about the change of rapidity dependence of the $S$ matrix from LO to running coupling, and from running coupling to full NLO cases. As we obtain the solution to the LO BK equation in Eq. (7), the exponent has quadratic rapidity dependence, $S \sim \exp \left(-\mathcal{O}\left(Y^{2}\right)\right)$. While this quadratic rapidity dependence is replaced by linear dependence, 


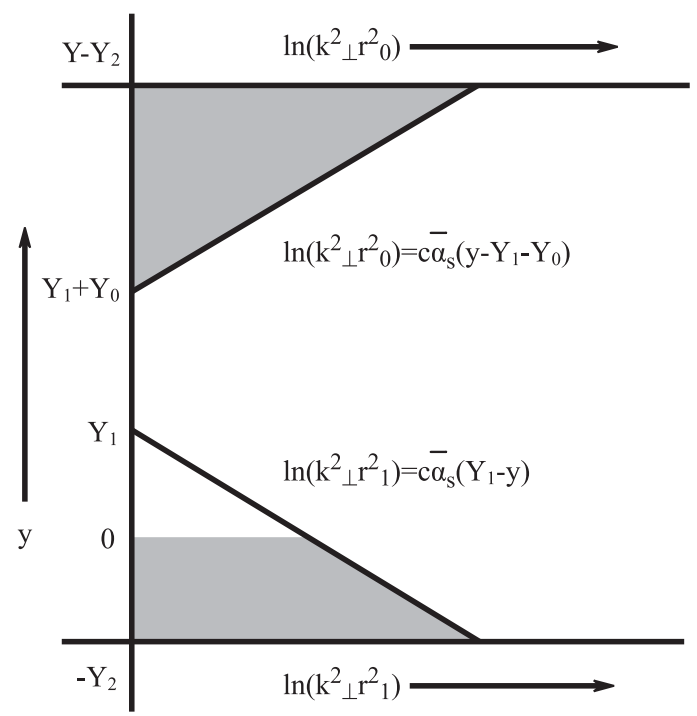

FIG. 3. The LO configuration in a general frame.

Eq. (15), once the running coupling corrections are taken into account, $\exp \left(-\mathcal{O}\left(Y^{2}\right)\right) \rightarrow \exp (-\mathcal{O}(Y))$. Further, when one includes full NLO contributions, the linear rapidity dependence of the $S$ matrix changes to rapidity raised to power of $3 / 2$ dependence, $\exp (-\mathcal{O}(Y)) \rightarrow$ $\exp \left(-\mathcal{O}\left(Y^{3 / 2}\right)\right)$. The global analysis shows that the gluon loop contributions (one part of the NLO corrections) compensate for part of the decrease of the rapidity made by quark loops. The compensation induces the rare fluctuations to become important again.

\section{EFFECTS OF RARE FLUCTUATIONS IN HIGH-ENERGY DIPOLE-DIPOLE SCATTERING IN A GENERAL FRAME}

To ensure that the results that we have obtained in the above section are independent of the frame choice, we study a scattering of a right-moving dipole of size $r_{0}$ and rapidity $Y-Y_{2}$ off a left-moving dipole of size $r_{1}$ and rapidity $-Y_{2}$ in a general frame. The frame and scattering pictures in the LO and NLO cases are given in Figs. 3 and 4, respectively.

\section{A. Leading-order case}

We let a right-moving dipole of size $r_{0}$ and rapidity $Y-Y_{2}$ scatter on a left-moving dipole of size $r_{1}$ and rapidity $-Y_{2}$ in a general frame. The scattering picture is illustrated in Fig. 3. We assume that the right-moving dipole is highly evolved, and that the left-moving dipole has the smaller rapidity. In this study, the $Y_{2} \leq \frac{1}{2}\left(Y-Y_{0}\right)$ is required for the sake of later calculation convenience, where $Y_{0}$ is the rapidity gap between two such dipoles in which the unitarity corrections begin to become important. In order to get a rare configuration, one has to restrict the evolution of both dipoles $r_{0}$ and $r_{1}$. For the dipole $r_{0}$, its evolution over

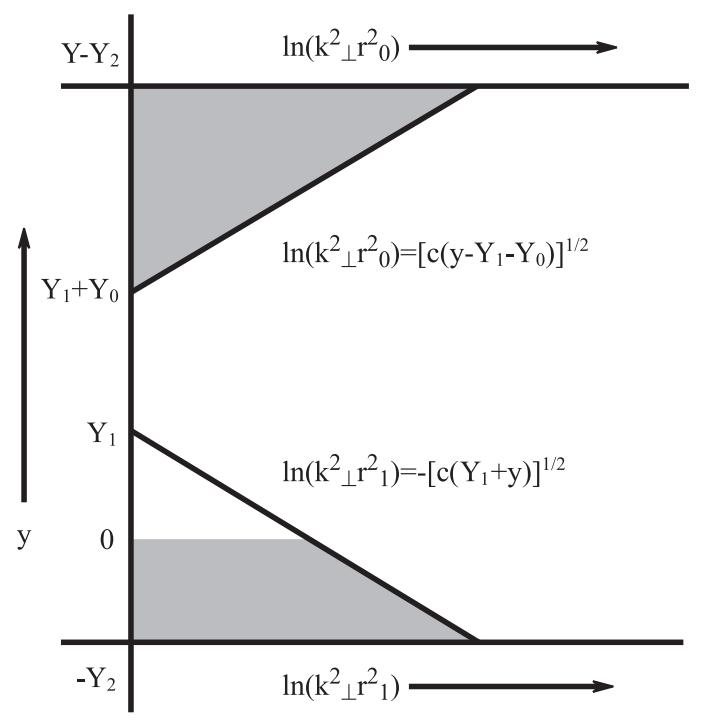

FIG. 4. The NLO configuration in a general frame.

the rapidity range $Y_{1}+Y_{0}<y<Y-Y_{2}$ (see the upper shaded triangle in Fig. 3) is suppressed as it was in Sec. IVA. In the lowest $Y_{0}+Y_{1}$ rapidity, the dipole $r_{0}$ has normal BFKL evolution. The unshaded triangle, $0<Y<Y_{1}$, is a saturation regime in which the dipole $r_{0}$ has evolved into a color glass condensate. $Y_{1}$ is an intermediate variable which will be determined by maximizing the $S$ matrix. Note that the line of the upper shaded triangle in Fig. 3,

$$
\ln \left(k_{\perp}^{2} r_{0}^{2}\right)=c \bar{\alpha}_{s}\left(y-Y_{1}-Y_{0}\right),
$$

is determined by the requirement that gluons locating on the right-hand side of that line cannot evolve by normal BFKL evolution into the shaded triangles. We wish to note that a similar approach is employed to determine the line of the lower shaded triangle in Fig. 3. For the dipole $r_{1}$, we have to strongly suppress the emission of those dipoles which can become of size $1 / Q_{s}$ through a normal evolution over the intermediate rapidity range $-Y_{2}<y<0$. This means that, at the time of scattering, the left-moving dipole system has no additional dipoles of size $\lambda r_{1}$ or larger, with $\lambda$ being a constant of order 1 . Based on this scattering picture, we can now estimate the $S$ matrix as [20]

$$
\begin{aligned}
S\left(r_{0}, r_{1}, Y\right)= & A_{R}\left(r_{0}, Y-Y_{0}-Y_{1}-Y_{2}\right) \\
& \times \mathcal{S}\left(r_{0}, r_{1}, Y_{0}+Y_{1}\right) A_{L}\left(r_{1}, Y_{2}\right),
\end{aligned}
$$

where $\mathcal{S}\left(r_{0}, r_{1}, Y_{0}+Y_{1}\right)$ is the $S$ matrix for the scattering of an elementary dipole $r_{1}$ on a color glass condensate which is evolved from the dipole $r_{0}$. It is a dipole-typical configuration interaction and can be computed by using the LO BK equation. After using Eq. (7), we obtain 


$$
\mathcal{S}\left(r_{0}, r_{1}, Y_{0}+Y_{1}\right)=\exp \left[-\frac{c}{2} \bar{\alpha}_{s}^{2} Y_{1}^{2}\right] S\left(r_{0}, Y_{0}\right)
$$

As usual, $A_{R}\left(r_{0}, Y-Y_{0}-Y_{1}-Y_{2}\right)$ and $A_{L}\left(r_{1}, Y_{2}\right)$ are the suppression factors which denote no emission from the two dipoles, and they are given in terms of the suppressions over the upper and lower shaded regions in Fig. 3,

$$
\begin{aligned}
& A_{R}\left(r_{0}, Y-Y_{2}-Y_{1}-Y_{0}\right) \\
& \quad=\exp \left[-\frac{c}{2} \bar{\alpha}_{s}^{2}\left(Y-Y_{2}-Y_{1}-Y_{0}\right)^{2}\right],
\end{aligned}
$$

and

$$
A_{L}\left(r_{1}, Y_{2}\right)=\exp \left[-\frac{c}{2} \bar{\alpha}_{s}^{2}\left(\left(Y_{1}+Y_{2}\right)^{2}-Y_{1}^{2}\right)\right] .
$$

Substituting Eqs. (37), (38), and (39) into Eq. (36), one obtains

$$
\begin{aligned}
S\left(r_{0}, r_{1}, Y\right)= & \exp \left[-\frac{c}{2} \bar{\alpha}_{s}^{2}\left[\left(Y-Y_{2}-Y_{1}-Y_{0}\right)^{2}\right.\right. \\
& \left.\left.+\left(Y_{1}+Y_{2}\right)^{2}\right]\right] S\left(r_{0}, Y_{0}\right) .
\end{aligned}
$$

Note that the $Y_{1}$ is a rapidity which describes the amount of evolution in the right-moving dipole, and it can be determined by maximizing the $S$ matrix through a minimization of the exponent of Eq. (40). So one can get the optimal value of $Y_{1}$ :

$$
Y_{1}=\frac{1}{2}\left(Y-Y_{0}\right)-Y_{2}
$$

Taking $Y_{1}$ into Eq. (40), one obtains the $S$ matrix

$$
S\left(r_{0}, r_{1}, Y\right)=\exp \left[-\frac{c}{4} \bar{\alpha}_{s}^{2}\left(Y-Y_{0}\right)^{2}\right] S\left(r_{0}, Y_{0}\right) .
$$

As expected, this result is exactly the same as the corresponding result (27) in the center of mass frame. We would like to point out that the right-hand size of Eq. (42) depends on $r_{1}$ through the rapidity $Y_{0}$.

\section{B. Next-to-leading-order case}

In the NLO case, the scattering picture in a general frame is illustrated in Fig. 4. The line of the upper shaded triangle in Fig. 4,

$$
\ln \left(k_{\perp}^{2} r_{0}^{2}\right)=\sqrt{c\left(y-Y_{1}-Y_{0}\right)},
$$

is determined by the requirement that gluons located on the right-hand side of that line cannot evolve by normal BFKL evolution into the shaded triangles. We wish to note that a similar approach is employed to determine the line of the lower shaded triangle in Fig. 4.

\section{In the case of running coupling}

We use the same approach as mentioned above to compute the $S$ matrix. Based on Eq. (36), we know that the suppression factors $A_{R}$ and $A_{L}$ need to be computed. Since the suppression process has a similar meaning as the survival probability of the parent dipoles in a normal BFKL evolution, it can be calculated by using the virtual term in the alternative form of Eq. (8) expressed in terms of the scattering amplitude $N$. One obtains

$$
\begin{aligned}
A_{R}\left(r_{0}, Y-Y_{0}-Y_{1}-Y_{2}\right)= & \exp \left[-\frac{N_{c} \mu}{c \pi \mu_{1}}\left(c \ln \left(\frac{1+\mu_{1} \sqrt{c\left(Y-Y_{2}-Y_{1}-Y_{0}\right)}}{1+\mu_{1} \ln \left(\frac{1}{r^{2} \Lambda^{2}}\right)}-\frac{1}{2}\right)\left(Y-Y_{2}-Y_{1}-Y_{0}\right)\right.\right. \\
& \left.\left.+\frac{\sqrt{c\left(Y-Y_{2}-Y_{1}-Y_{0}\right)}}{\mu_{1}}-\frac{1}{\mu_{1}^{2}} \ln \left(1+\mu_{1} \sqrt{c\left(Y-Y_{2}-Y_{1}-Y_{0}\right)}\right)\right)\right]
\end{aligned}
$$

and

$$
\begin{aligned}
A_{L}\left(r_{1}, Y_{2}\right)= & \exp \left\{-\frac{N_{c} \mu}{c \pi \mu_{1}}\left[c \ln \left(\frac{1+\mu_{1} \sqrt{c\left(Y_{1}+Y_{2}\right)}}{1+\mu_{1} \ln \left(\frac{1}{r^{2} \Lambda^{2}}\right)}-\frac{1}{2}\right)\left(Y_{1}+Y_{2}\right)+\frac{\sqrt{c\left(Y_{1}+Y_{2}\right)}}{\mu_{1}}-\frac{1}{\mu_{1}^{2}} \ln \left(1+\mu_{1} \sqrt{c\left(Y_{1}+Y_{2}\right)}\right)\right.\right. \\
& \left.\left.-c \ln \left(\frac{1+\mu_{1} \sqrt{c Y_{1}}}{1+\mu_{1} \ln \left(\frac{1}{r^{2} \Lambda^{2}}\right)}-\frac{1}{2}\right) Y_{1}-\frac{\sqrt{c Y_{1}}}{\mu_{1}}+\frac{1}{\mu_{1}^{2}} \ln \left(1+\mu_{1} \sqrt{c Y_{1}}\right)\right]\right\}
\end{aligned}
$$

The $S$ matrix for the dipole-typical configuration interaction in the running coupling case is derived in Eq. (15). By using Eq. (15), the $\mathcal{S}\left(r_{0}, r_{1}, Y_{0}+Y_{1}\right)$ in Eq. (36) can be written as

$$
\mathcal{S}\left(r_{0}, r_{1}, Y_{0}+Y_{1}\right)=\exp \left\{-\frac{N_{c} \mu}{c \pi \mu_{1}}\left[c \ln \left(\frac{1+\mu_{1} \sqrt{c Y_{1}}}{1+\mu_{1} \ln \left(\frac{1}{r^{2} \Lambda^{2}}\right)}-\frac{1}{2}\right) Y_{1}+\frac{\sqrt{c Y_{1}}}{\mu_{1}}-\frac{1}{\mu_{1}^{2}} \ln \left(1+\mu_{1} \sqrt{c Y_{1}}\right)\right]\right\} S\left(r_{0}, Y_{0}\right) .
$$


Combining Eqs. (44), (45), and (46), one gets

$$
\begin{aligned}
S\left(r_{0}, r, Y\right)= & A_{R}\left(r_{0}, Y-Y_{0}-Y_{1}-Y_{2}\right) \mathcal{S}\left(r_{0}, r_{1}, Y_{0}+Y_{1}\right) A_{L}\left(r_{1}, Y_{2}\right) \\
= & \exp \left\{-\frac{N_{c} \mu}{c \pi \mu_{1}}\left[c \ln \left(\frac{1+\mu_{1} \sqrt{c\left(Y-Y_{2}-Y_{1}-Y_{0}\right)}}{1+\mu_{1} \ln \left(\frac{1}{r^{2} \Lambda^{2}}\right)}-\frac{1}{2}\right)\left(Y-Y_{2}-Y_{1}-Y_{0}\right)+\frac{\sqrt{c\left(Y-Y_{2}-Y_{1}-Y_{0}\right)}}{\mu_{1}}\right.\right. \\
& -\frac{1}{\mu_{1}^{2}} \ln \left(1+\mu_{1} \sqrt{c\left(Y-Y_{2}-Y_{1}-Y_{0}\right)}\right)+c \ln \left(\frac{1+\mu_{1} \sqrt{c\left(Y_{1}+Y_{2}\right)}}{1+\mu_{1} \ln \left(\frac{1}{r^{2} \Lambda^{2}}\right)}-\frac{1}{2}\right)\left(Y_{1}+Y_{2}\right)+\frac{\sqrt{c\left(Y_{1}+Y_{2}\right)}}{\mu_{1}} \\
& \left.\left.-\frac{1}{\mu_{1}^{2}} \ln \left(1+\mu_{1} \sqrt{c\left(Y_{1}+Y_{2}\right)}\right)\right]\right\} S\left(r_{0}, Y_{0}\right) .
\end{aligned}
$$

The maximum value of the $S$ matrix can be determined in the same way as in the leading order case. One can get the optimal value of $Y_{1}$ as in Eq. (41). Substituting Eq. (41) into Eq. (47), the final $S$ matrix is

$S\left(r_{0}, r_{1}, Y\right)=\exp \left\{-\frac{N_{c} \mu}{c \pi \mu_{1}}\left[\ln ^{2}\left(\frac{Q_{S}^{2}(Y)}{\Lambda^{2}}\right) \ln \left(\frac{1+\frac{\mu_{1}}{\sqrt{2}} \ln \left(\frac{Q_{S}^{2}(Y)}{\Lambda^{2}}\right)}{1+\mu_{1} \ln \left(\frac{1}{r^{2} \Lambda^{2}}\right)}-\frac{1}{2}\right)+\frac{\sqrt{2} \ln \left(\frac{Q_{S}^{2}(Y)}{\Lambda^{2}}\right)}{\mu_{1}}-\frac{2}{\mu_{1}^{2}} \ln \left(1+\frac{\mu_{1}}{\sqrt{2}} \ln \left(\frac{Q_{S}^{2}(Y)}{\Lambda^{2}}\right)\right)\right]\right\} S\left(r_{0}, Y_{0}\right)$,

which is exactly the same as the one, Eq. (30), in the center of mass frame.

\section{In the case of full next-to-leading order}

Since we have already derived the effect of rare fluctuations on top of running coupling, it is now easy to transform the corresponding formalisms to the full next-to-leading case by changing only the corresponding running coupling $S$ matrix to the $S$ matrix of full next-to-leading corrections. Using Eq. (22), we obtain

$$
\begin{aligned}
A_{R}\left(r, Y-Y_{0}-Y_{1}-Y_{2}\right)= & \exp \left[-\frac{N_{c} \mu}{c \pi \mu_{1}}\left(\frac{2 C_{r}}{3}\left(c\left(Y-Y_{0}-Y_{1}-Y_{2}\right)\right)^{3 / 2}+c\left(Y-Y_{0}-Y_{1}-Y_{2}\right)\right.\right. \\
& \times \ln \frac{\left(r^{2} \Lambda^{2}\right)^{C_{r}}+\left(r^{2} \Lambda^{2}\right)^{C_{r}} \mu_{1}\left(c\left(Y-Y_{0}-Y_{1}-Y_{2}\right)\right)^{1 / 2}}{1+\mu_{1} \ln \frac{1}{\left(r^{2} \Lambda^{2}\right)}}+\frac{1}{\mu_{1}}\left(c\left(Y-Y_{0}-Y_{1}-Y_{2}\right)\right)^{1 / 2} \\
& \left.\left.-\frac{1}{\mu_{1}^{2}} \ln \left(1+\mu_{1}\left(c\left(Y-Y_{0}-Y_{1}-Y_{2}\right)\right)^{1 / 2}\right)\right)\right]
\end{aligned}
$$

and

$$
\begin{aligned}
& A_{L}\left(r_{1}, Y_{2}\right)=\exp \left[-\frac{N_{c} \mu}{c \pi \mu_{1}}\left(\frac{2 C_{r}}{3}\left(c\left(Y_{1}+Y_{2}\right)\right)^{3 / 2}+c\left(Y_{1}+Y_{2}\right) \ln \frac{\left(r^{2} \Lambda^{2}\right)^{C_{r}}+\left(r^{2} \Lambda^{2}\right)^{C_{r}} \mu_{1}\left(c\left(Y_{1}+Y_{2}\right)\right)^{1 / 2}}{1+\mu_{1} \ln \frac{1}{\left(r^{2} \Lambda^{2}\right)}}+\frac{1}{\mu_{1}}\left(c\left(Y_{1}+Y_{2}\right)\right)^{1 / 2}\right.\right. \\
& -\frac{1}{\mu_{1}^{2}} \ln \left(1+\mu_{1}\left(c\left(Y_{1}+Y_{2}\right)\right)^{1 / 2}\right)-\frac{2 C_{r}}{3}\left(c Y_{1}\right)^{3 / 2}-c Y_{1} \ln \frac{\left(r^{2} \Lambda^{2}\right)^{C_{r}}+\left(r^{2} \Lambda^{2}\right)^{C_{r}} \mu_{1}\left(c Y_{1}\right)^{1 / 2}}{1+\mu_{1} \ln \frac{1}{\left(r^{2} \Lambda^{2}\right)}} \\
& \left.\left.-\frac{1}{\mu_{1}}\left(c Y_{1}\right)^{1 / 2}+\frac{1}{\mu_{1}^{2}} \ln \left(1+\mu_{1}\left(c Y_{1}\right)^{1 / 2}\right)\right)\right] \text {. }
\end{aligned}
$$

Similarly, the $S$ matrix for the scattering of an elementary dipole on a color glass condensate state is

$$
\begin{aligned}
S\left(r_{0}, r_{1}, Y_{0}+Y_{1}\right)= & \exp \left[-\frac{N_{c} \mu}{c \pi \mu_{1}}\left(\frac{2 C_{r}}{3}\left(c Y_{1}\right)^{3 / 2}+c Y_{1} \ln \frac{\left(r^{2} \Lambda^{2}\right)^{C_{r}}+\left(r^{2} \Lambda^{2}\right)^{C_{r}} \mu_{1}\left(c Y_{1}\right)^{1 / 2}}{1+\mu_{1} \ln \frac{1}{\left(r^{2} \Lambda^{2}\right)}}\right.\right. \\
& \left.\left.+\frac{1}{\mu_{1}}\left(c Y_{1}\right)^{1 / 2}-\frac{1}{\mu_{1}^{2}} \ln \left(1+\mu_{1}\left(c Y_{1}\right)^{1 / 2}\right)\right)\right] S\left(r_{0}, Y_{0}\right) .
\end{aligned}
$$


Thus,

$$
\begin{aligned}
S\left(r_{0}, r_{1}, Y\right)= & A_{R}\left(r_{0}, Y-Y_{0}-Y_{1}-Y_{2}\right) S\left(r_{0}, r_{1}, Y_{0}+Y_{1}\right) A_{L}\left(r_{1}, Y_{2}\right) \\
= & \exp \left[-\frac{N_{c} \mu}{c \pi \mu_{1}}\left(\frac{2 C_{r}}{3}\left(c\left(Y-Y_{0}-Y_{1}-Y_{2}\right)\right)^{3 / 2}+c\left(Y-Y_{0}-Y_{1}-Y_{2}\right)\right.\right. \\
& \times \ln \frac{\left(r^{2} \Lambda^{2}\right)^{C_{r}}+\left(r^{2} \Lambda^{2}\right)^{C_{r}} \mu_{1}\left(c\left(Y-Y_{0}-Y_{1}-Y_{2}\right)\right)^{1 / 2}}{1+\mu_{1} \ln \frac{1}{\left(r^{2} \Lambda^{2}\right)}}+\frac{1}{\mu_{1}}\left(c\left(Y-Y_{0}-Y_{1}-Y_{2}\right)\right)^{1 / 2} \\
& \left.-\frac{1}{\mu_{1}^{2}} \ln \left(1+\mu_{1}\left(c\left(Y-Y_{0}-Y_{1}-Y_{2}\right)\right)^{1 / 2}\right)\right)-\frac{N_{c} \mu}{2 c \pi \mu_{1}}\left(\frac{2 C_{r}}{3}\left(c\left(Y_{1}+Y_{2}\right)\right)^{3 / 2}+c\left(Y_{1}+Y_{2}\right)\right. \\
& \left.\times \ln \frac{\left(r^{2} \Lambda^{2}\right)^{C_{r}}+\left(r^{2} \Lambda^{2}\right)^{C_{r}} \mu_{1}\left(c\left(Y_{1}+Y_{2}\right)\right)^{1 / 2}}{1+\mu_{1} \ln \frac{1}{\left(r^{2} \Lambda^{2}\right)}}+\frac{1}{\mu_{1}}\left(c\left(Y_{1}+Y_{2}\right)\right)^{1 / 2}-\frac{1}{\mu_{1}^{2}} \ln \left(1+\mu_{1}\left(c\left(Y_{1}+Y_{2}\right)\right)^{1 / 2}\right)\right] S\left(r_{0}, Y_{0}\right) .
\end{aligned}
$$

Substituting the optimal value of $Y_{1}$ into Eq. (52), the maximum value of the $S$ matrix is

$$
\begin{aligned}
& S\left(r_{0}, r_{1}, Y\right)=\exp \left[-\frac{N_{c} \mu}{2 c \pi \mu_{1}}\left(\frac{2 C_{r}}{3} 2\left(c \frac{Y-Y_{0}}{2}\right)^{3 / 2}+2 c \frac{\left(Y-Y_{0}\right)}{2} \ln \frac{\left(r^{2} \Lambda^{2}\right)^{C_{r}}+\left(r^{2} \Lambda^{2}\right)^{C_{r}} \mu_{1}\left(c \frac{Y-Y_{0}}{2}\right)^{1 / 2}}{1+\mu_{1} \ln \frac{1}{\left(r^{2} \Lambda^{2}\right)}}+\frac{2}{\mu_{1}}\left(c \frac{Y-Y_{0}}{2}\right)^{1 / 2}\right.\right. \\
& \left.\left.-\frac{2}{\mu_{1}^{2}} \ln \left(1+\mu_{1}\left(c \frac{Y-Y_{0}}{2}\right)^{1 / 2}\right)\right)\right] S\left(r_{0}, Y_{0}\right) \\
& =\exp \left[-\frac{N_{c} \mu}{2 c \pi \mu_{1}}\left(\frac{1}{\sqrt{2}} \frac{2 C_{r}}{3} \ln ^{3} \frac{Q_{s}^{2}(Y)}{\Lambda^{2}}+\ln ^{2} \frac{Q_{s}^{2}(Y)}{\Lambda^{2}} \ln \frac{\left(r^{2} \Lambda^{2}\right)^{C_{r}}+\left(r^{2} \Lambda^{2}\right)^{C_{r}} \frac{\mu_{1}}{\sqrt{2}} \ln \frac{Q_{s}^{2}(Y)}{\Lambda^{2}}}{1+\mu_{1} \ln \frac{1}{\left(r^{2} \Lambda^{2}\right)}}\right.\right. \\
& \left.\left.+\frac{\sqrt{2}}{\mu_{1}} \ln \frac{Q_{s}^{2}(Y)}{\Lambda^{2}}-\frac{2}{\mu_{1}^{2}} \ln \left(1+\frac{\mu_{1}}{\sqrt{2}} \ln \frac{Q_{s}^{2}(Y)}{\Lambda^{2}}\right)\right)\right] S\left(r_{0}, Y_{0}\right)
\end{aligned}
$$

which is exactly the same as the corresponding result (34) in the center of mass frame.

Through the calculations that we have done above, we know that the results of Eqs. (27), (30), and (34) are independent of the frame choice. The exponential factors of the $S$ matrices are twice and $\sqrt{2}$ as large as the results which emerge when the rare fluctuation effects are taken into account in the LO and full NLO cases, respectively. These findings indicate that the rare fluctuation effects are important in the LO and full NLO cases.

\section{ACKNOWLEDGMENTS}

This work is supported by the National Natural Science Foundation of China under Grants No. 11765005, No. 11305040, No. 11847152, and No. 11775097; the Department of Science and Technology of Guizhou Province under Grants No. [2018]1023, No. [2019]5668 and No. [2019]5103; Qian Kehe Platform Talents No. [2017]5736-013 and No. [2017]5736-027; the
Department of Education of Guizhou Province under Grant No. KY[2017]004; and the National Key Research and Development Program of China under Grants No. 2015 CB856905 and No. CCNU18ZDPY04.

\section{APPENDIX: CALCULATE THE ANALYTIC SOLUTION OF THE FULL NLO BK EVOLUTION EQUATION}

In this appendix, we give the details of the derivation of the analytic solutions to the full NLO BK evolution equation in two different approaches: one is developed by us and the other is used in Ref. [31]. Then we compare them with each other.

The full NLO BK evolution equation is given by Eq. (17). In this work, we analytically solve the equation in the saturation region where the $S$ matrix is very small. Therefore, the quadratic and cubic terms of the $S$ matrix in Eq. (17) are neglected. Thus, Eq. (17) is reduced to a simple linear equation,

$$
\frac{\partial S(r, Y)}{\partial Y}=-\int \mathrm{d}^{2} r_{1} \frac{\bar{\alpha}_{s}}{2 \pi} \frac{r^{2}}{r_{1}^{2} r_{2}^{2}}\left\{1+\frac{\bar{\alpha}_{s}}{4}\left[b \ln r^{2} \mu^{2}-b \frac{r_{1}^{2}-r_{2}^{2}}{r^{2}} \ln \frac{r_{1}^{2}}{r_{2}^{2}}+\frac{67}{9}-\frac{\pi^{2}}{3}-\frac{10 N_{\mathrm{f}}}{9 N_{\mathrm{c}}}-2 \ln \frac{r_{1}^{2}}{r^{2}} \ln \frac{r_{2}^{2}}{r^{2}}\right]\right\} S(r, Y)
$$


In the above equation, we have used the notation $\mathbf{r}=x_{\perp}-y_{\perp}, \mathbf{r}_{1}=x_{\perp}-z_{\perp}$, and $\mathbf{r}_{2}=z_{\perp}-y_{\perp}$. The running coupling terms in Eq. (A1) are scale dependent, and a scheme developed in Ref. [37] is employed to rewrite the running coupling part. If we replace all terms proportional to $b$ with the Balitsky running coupling, Eq. (A1) can be rewritten as

$\frac{\partial S(r, Y)}{\partial Y}=-\int \mathrm{d}^{2} r_{1} \frac{\bar{\alpha}_{S}\left(r^{2}\right)}{2 \pi}\left\{\frac{r^{2}}{r_{1}^{2} r_{2}^{2}}+\frac{1}{r_{1}^{2}}\left[\frac{\alpha_{s}\left(r_{1}^{2}\right)}{\alpha_{s}\left(r_{2}^{2}\right)}-1\right]+\frac{1}{r_{2}^{2}}\left[\frac{\alpha_{s}\left(r_{2}^{2}\right)}{\alpha_{s}\left(r_{1}^{2}\right)}-1\right]+\frac{\bar{\alpha}_{s}\left(r^{2}\right)}{4} \frac{r^{2}}{r_{1}^{2} r_{2}^{2}}\left[\frac{67}{9}-\frac{\pi^{2}}{3}-\frac{10 N_{f}}{9 N_{c}}-2 \ln \frac{r_{1}^{2}}{r^{2}} \ln \frac{r_{2}^{2}}{r^{2}}\right]\right\} S(r, Y)$.

For solving Eq. (A2), we can choose the saturation region to be either $1 / Q_{s} \ll\left|\mathbf{r}_{\mathbf{1}}\right| \ll|\mathbf{r}|,\left|\mathbf{r}_{\mathbf{2}}\right| \sim|\mathbf{r}|$ or $1 / Q_{s} \ll\left|\mathbf{r}_{\mathbf{2}}\right| \ll|\mathbf{r}|,\left|\mathbf{r}_{\mathbf{1}}\right| \sim|\mathbf{r}|$, as mentioned in Sec. III B. Under this saturation condition, Eq. (A2) can be simplified to

$$
\begin{aligned}
\frac{\partial S(r, Y)}{\partial Y} & =-2 \int_{1 / Q_{s}^{2}}^{r^{2}} \mathrm{~d}^{2} r_{1} \frac{\bar{\alpha}_{s}\left(r^{2}\right)}{2 \pi}\left[\frac{1}{r_{1}^{2}} \frac{\alpha_{s}\left(r_{1}^{2}\right)}{\alpha_{s}\left(r^{2}\right)}+\frac{1}{r^{2}}\left(\frac{\alpha_{s}\left(r^{2}\right)}{\alpha_{s}\left(r_{1}^{2}\right)}-1\right)+\frac{\bar{\alpha}_{s}\left(r^{2}\right)}{4} \frac{1}{r_{1}^{2}}\left(\frac{67}{9}-\frac{\pi^{2}}{3}-\frac{10 N_{f}}{9 N_{c}}-2 \ln \frac{r_{1}^{2}}{r^{2}} \ln \frac{r_{2}^{2}}{r^{2}}\right)\right] S(r, Y) \\
& \simeq-2 \int_{1 / Q_{s}^{2}}^{r^{2}} \mathrm{~d}^{2} r_{1}\left[\frac{\bar{\alpha}_{s}\left(r_{1}^{2}\right)}{2 \pi r_{1}^{2}}+\frac{\bar{\alpha}_{s}^{2}\left(r^{2}\right)}{8 \pi r_{1}^{2}}\left(\frac{67}{9}-\frac{\pi^{2}}{3}-\frac{10 N_{f}}{9 N_{c}}\right)\right] S(r, Y) \\
& =-2 \frac{1}{2 \pi} \int_{1 / Q_{s}^{2}}^{r^{2}} \mathrm{~d}^{2} r_{1}\left[\frac{\bar{\alpha}_{s}\left(r_{1}^{2}\right)}{r_{1}^{2}}+\frac{\bar{\alpha}_{s}^{2}\left(r^{2}\right)}{4} \frac{1}{r_{1}^{2}}\left(\frac{67}{9}-\frac{\pi^{2}}{3}-\frac{10 N_{f}}{9 N_{c}}\right)\right] S(r, Y) .
\end{aligned}
$$

For the running coupling and saturation momentum mentioned in the above equation, we use Eqs. (13) and (16). Substituting Eq. (14) into Eq. (A3), we can get

$$
\begin{aligned}
\frac{\partial S(r, Y)}{\partial Y} & =-\frac{N_{c}}{\pi} \int_{\ln 1 / Q_{s}^{2}}^{\ln r^{2}} \mathrm{~d} \ln r_{1}^{2}\left[\frac{\mu}{1+\mu_{1} \ln \left(\frac{1}{r_{1}^{2} \Lambda^{2}}\right)}+\frac{N_{c}}{\pi} \frac{\alpha_{s}\left(r^{2}\right)}{4}\left(\frac{67}{9}-\frac{\pi^{2}}{3}-\frac{10 N_{f}}{9 N_{c}}\right)\right] S(r, Y) \\
& =\left\{-\frac{N_{c} \mu}{\pi \mu_{1}}\left[\ln \left[1+\mu_{1} \ln \frac{Q_{s}^{2}(Y)}{\Lambda^{2}}\right]-\ln \left(1+\mu_{1} \ln \frac{1}{r^{2} \Lambda^{2}}\right)\right]+\frac{N_{c}^{2}}{\pi^{2}} \frac{\alpha_{s}\left(r^{2}\right)}{4}\left(\frac{67}{9}-\frac{\pi^{2}}{3}-\frac{10 N_{f}}{9 N_{c}}\right) \ln \left(Q_{s}^{2} r^{2}\right)\right\} S(r, Y) .
\end{aligned}
$$

By integrating Eq. (A4) over $Y$, we can obtain the analytic solution [21]

$$
\begin{aligned}
& S(r, Y)=\exp \left[-\frac{N_{c} \mu}{c \pi \mu_{1}}\left(\frac{2 C_{r}}{3} \ln ^{3} \frac{Q_{s}^{2}(Y)}{\Lambda^{2}}+\ln ^{2} \frac{Q_{s}^{2}(Y)}{\Lambda^{2}} \ln \frac{\left(r^{2} \Lambda^{2}\right)^{C_{r}}+\left(r^{2} \Lambda^{2}\right)^{C_{r}} \mu_{1} \ln \frac{Q_{s}^{2}(Y)}{\Lambda^{2}}}{1+\mu_{1} \ln \frac{1}{\left(r^{2} \Lambda^{2}\right)}}+\frac{1}{\mu_{1}} \ln \frac{Q_{s}^{2}(Y)}{\Lambda^{2}}\right.\right. \\
& \left.\left.-\frac{1}{\mu_{1}^{2}} \ln \left(1+\mu_{1} \ln \frac{Q_{s}^{2}(Y)}{\Lambda^{2}}\right)\right)\right] S\left(r, Y_{0}\right)
\end{aligned}
$$

with $C_{r}=\alpha_{s}^{2}\left(r^{2}\right) N_{c} \mu_{1}\left(67 / 9-\pi^{2} / 3-10 N_{f} / 9 N_{c}\right) / 4 \pi \mu$. Note that Eq. (A5) is the same as Eq. (22).

On the other hand, the authors in Ref. [31] solved Eq. (A1) by taking the integration in the polar coordinate. To perform the integration, they rewrote Eq. (A1) as

$$
\frac{\partial S(r, Y)}{\partial Y}=-\frac{\bar{\alpha}_{s}}{2 \pi} K^{\operatorname{Con}}\left(Q_{s}, r\right) S(r, Y)
$$

with

$$
K^{\mathrm{Con}}\left(Q_{s}, r\right)=\int \mathrm{d}^{2} r_{1} \frac{r^{2}}{r_{1}^{2} r_{2}^{2}}\left\{1+\frac{\bar{\alpha}_{s}}{4}\left[b \ln r^{2} \mu^{2}-b \frac{r_{1}^{2}-r_{2}^{2}}{r^{2}} \ln \frac{r_{1}^{2}}{r_{2}^{2}}+\frac{67}{9}-\frac{\pi^{2}}{3}-\frac{10 N_{\mathrm{f}}}{9 N_{\mathrm{c}}}-2 \ln \frac{r_{1}^{2}}{r^{2}} \ln \frac{r_{2}^{2}}{r^{2}}\right]\right\} .
$$

Then the integration of Eq. (A7) can be divided into three parts:

$$
\begin{aligned}
K^{\mathrm{Con}}\left(Q_{s}, r\right)= & \int \mathrm{d}^{2} r_{1} \frac{r^{2}}{r_{1}^{2} r_{2}^{2}}\left\{1+\frac{\bar{\alpha}_{s}}{4}\left[b \ln r^{2} \mu^{2}+\frac{67}{9}-\frac{\pi^{2}}{3}-\frac{10 N_{\mathrm{f}}}{9 N_{\mathrm{c}}}\right]\right\}+\int \mathrm{d}^{2} r_{1} \frac{r^{2}}{r_{1}^{2} r_{2}^{2}} \frac{\bar{\alpha}_{s}}{4} b \frac{r_{2}^{2}-r_{1}^{2}}{r^{2}} \ln \frac{r_{1}^{2}}{r_{2}^{2}} \\
& -\int \mathrm{d}^{2} r_{1} \frac{r^{2}}{r_{1}^{2} r_{2}^{2}} \frac{\bar{\alpha}_{s}}{2} \ln \frac{r_{1}^{2}}{r^{2}} \ln \frac{r_{2}^{2}}{r^{2}} .
\end{aligned}
$$


Following Ref. [31], we define $R_{0}^{2}=1 / Q_{s}^{2} r^{2}, R_{1}^{2}=1-1 / Q_{s}^{2} r^{2}$, and $R^{2}=r_{1}^{2} / r^{2}$. The three integral terms on the right-hand side of Eq. (A8) can be rewritten, respectively, as [31]

$$
\begin{gathered}
I_{1}=\frac{1}{2} \int_{R_{0}^{2}}^{R_{1}^{2}} \frac{d R^{2}}{R^{2}} \int_{0}^{2 \pi} \frac{1}{1+R^{2}-2 R \cos \theta} d \theta\left\{1+\frac{\bar{\alpha}_{s}}{4}\left[b \ln r^{2} \mu^{2}+\frac{67}{9}-\frac{\pi^{2}}{3}-\frac{10 N_{\mathrm{f}}}{9 N_{\mathrm{c}}}\right]\right\} \\
I_{2}=\frac{\bar{\alpha}_{s}}{4} b \int_{R_{0}^{2}}^{R_{1}^{2}} \frac{d R^{2}}{R^{2}} \int_{0}^{2 \pi} \ln \left(\frac{R^{2}}{1+R^{2}-2 R \cos \theta}\right) d \theta \\
I_{3}=-\frac{\bar{\alpha}_{s}}{4} \int_{R_{0}^{2}}^{R_{1}^{2}} \frac{d R^{2}}{R^{2}} \ln \left(R^{2}\right) \int_{0}^{2 \pi} \frac{1}{1+R^{2}-2 R \cos \theta} \ln \left(1+R^{2}-2 R \cos \theta\right) d \theta
\end{gathered}
$$

In the saturation region, one performs integrations on Eqs. (A9), (A10), and (A11) and gets [31]

$$
\begin{aligned}
& I_{1}=\frac{1}{2} \int_{R_{0}^{2}}^{R_{1}^{2}} \frac{d R^{2}}{R^{2}} 2 \pi \frac{1}{1-R^{2}}\left\{1+\frac{\bar{\alpha}_{s}}{4}\left[b \ln r^{2} \mu^{2}+\frac{67}{9}-\frac{\pi^{2}}{3}-\frac{10 N_{\mathrm{f}}}{9 N_{\mathrm{c}}}\right]\right\} \\
& =\pi\left\{1+\frac{\bar{\alpha}_{s}}{4}\left[b \ln r^{2} \mu^{2}+\frac{67}{9}-\frac{\pi^{2}}{3}-\frac{10 N_{\mathrm{f}}}{9 N_{\mathrm{c}}}\right]\right\} \int_{R_{0}^{2}}^{R_{1}^{2}} \frac{d R^{2}}{R^{2}\left(1-R^{2}\right)} \\
& =\pi\left\{1+\frac{\bar{\alpha}_{s}}{4}\left[b \ln r^{2} \mu^{2}+\frac{67}{9}-\frac{\pi^{2}}{3}-\frac{10 N_{\mathrm{f}}}{9 N_{\mathrm{c}}}\right]\right\}\left[\ln \left(\frac{R_{1}^{2}}{R_{0}^{2}}\right)-\ln \left(\frac{1-R_{0}^{2}}{1-R_{1}^{2}}\right)\right] \\
& =2 \pi \ln \left(Q_{s}^{2} r^{2}\right)\left\{1+\frac{\bar{\alpha}_{s}}{4}\left[b \ln r^{2} \mu^{2}+\frac{67}{9}-\frac{\pi^{2}}{3}-\frac{10 N_{\mathrm{f}}}{9 N_{\mathrm{c}}}\right]\right\} \text {, } \\
& I_{2}=\frac{\bar{\alpha}_{s}}{4} b \int_{R_{0}^{2}}^{R_{1}^{2}} \frac{d R^{2}}{R^{2}} \int_{0}^{2 \pi} \ln \left(\frac{R^{2}}{1+R^{2}-2 R \cos \theta}\right) d \theta \\
& =\frac{\bar{\alpha}_{s}}{4} b \int_{R_{0}^{2}}^{R_{1}^{2}} \frac{d R^{2}}{R^{2}} \int_{0}^{2 \pi}\left[\ln R^{2}+2 \sum_{n=1}^{\infty} \frac{\cos (n \theta)}{n} R^{n}\right] d \theta \\
& =\frac{\bar{\alpha}_{s}}{4} b \int_{R_{0}^{2}}^{R_{1}^{2}} \frac{d R^{2}}{R^{2}} 2 \pi \ln R^{2} \\
& =\pi \frac{\bar{\alpha}_{s}}{4} b \ln ^{2}\left(\frac{R_{1}^{2}}{R_{0}^{2}}\right) \\
& =\pi \frac{\bar{\alpha}_{s}}{4} b \ln ^{2}\left(\frac{1-1 / Q_{s}^{2} r^{2}}{1 / Q_{s}^{2} r^{2}}\right) \\
& \simeq \pi \frac{\bar{\alpha}_{s}}{4} b \ln ^{2}\left(Q_{s}^{2} r^{2}\right), \\
& I_{3}=-\frac{\bar{\alpha}_{s}}{4} \int_{R_{0}^{2}}^{R_{1}^{2}} \frac{d R^{2}}{R^{2}} \ln \left(R^{2}\right) \int_{0}^{2 \pi} \frac{1}{1+R^{2}-2 R \cos \theta}\left(-2 \sum_{n=1}^{\infty} \frac{\cos (n \theta)}{n} R^{n}\right) d \theta \\
& =-\frac{\bar{\alpha}_{S}}{4} \int_{R_{0}^{2}}^{R_{1}^{2}} d R^{2} \frac{\ln \left(R^{2}\right)}{R^{2}\left(1-R^{2}\right)} \sum_{n=1}^{\infty} \frac{R^{2 n}}{n} \\
& =-\pi \bar{\alpha}_{s} \int_{R_{0}^{2}}^{R_{1}^{2}} d R^{2} \frac{\ln \left(R^{2}\right) \ln \left(1-R^{2}\right)}{R^{2}\left(1-R^{2}\right)} \\
& =-2 \pi \zeta(3) \text {. }
\end{aligned}
$$


Substituting Eqs. (A9), (A10), and (A11) into Eq. (A8), the evolution equation becomes

$$
\frac{\partial S(r, Y)}{\partial Y}=\left\{-\bar{\alpha}_{s}\left[1+\frac{\bar{\alpha}_{s}}{4}\left(b \ln r^{2} \mu^{2}+\frac{67}{9}-\frac{\pi^{2}}{3}-\frac{10 N_{\mathrm{f}}}{9 N_{\mathrm{c}}}\right)\right] \ln \left(Q_{s}^{2} r^{2}\right)-\frac{\bar{\alpha}_{s}^{2}}{8} b \ln ^{2}\left(Q_{s}^{2} r^{2}\right)+\bar{\alpha}_{s} \zeta(3)\right\} S(r, Y) .
$$

As was done in Ref. [31], we replace $\mu^{2}$ with $Q_{s}^{2}$, and Eq. (A15) reduces to

$$
\mathrm{d} \ln S(r, Y)=\left\{-\bar{\alpha}_{s}\left(Q_{s}\right)\left[1+\frac{3 \bar{\alpha}_{s}\left(Q_{s}\right)}{8} b \ln \left(Q_{s}^{2} r^{2}\right)+\bar{\alpha}_{s}\left(Q_{s}\right)\left(\frac{67}{36}-\frac{\pi^{2}}{12}-\frac{5 N_{\mathrm{f}}}{18 N_{\mathrm{c}}}\right)\right] \ln \left(Q_{s}^{2} r^{2}\right)+\bar{\alpha}_{s}\left(Q_{s}\right) \zeta(3)\right\} \mathrm{d} Y .
$$

We take the same form of the saturation momentum as Ref. [31],

$$
\ln \left(Q_{s}^{2}(Y) / Q_{0}^{2}\right)=\ln \left(Q_{s}^{2}(Y) r^{2}\right)-\ln \left(Q_{0}^{2} r^{2}\right)=\bar{\alpha}_{s}\left(Q_{s}\right) \times \rho \times\left(Y-Y_{0}\right) ;
$$

then we can rewrite Eq. (A16) as

$$
\mathrm{d} \ln S(r, Y)=\left\{-\bar{\alpha}_{s}\left(Q_{s}\right)\left[1+\frac{3 \bar{\alpha}_{s}\left(Q_{s}\right)}{8} b \ln \left(Q_{s}^{2} r^{2}\right)+\bar{\alpha}_{s}\left(Q_{s}\right)\left(\frac{67}{36}-\frac{\pi^{2}}{12}-\frac{5 N_{\mathrm{f}}}{18 N_{\mathrm{c}}}\right)\right] \ln \left(Q_{s}^{2} r^{2}\right)+\bar{\alpha}_{s}\left(Q_{s}\right) \zeta(3)\right\} \frac{\mathrm{d} \ln \left(Q_{s}^{2} r^{2}\right)}{\bar{\alpha}_{s}\left(Q_{s}\right) \rho} .
$$

One can easily get the solution of the above equation as [31]

$$
S(r, Y)=\exp \left\{-\frac{1}{2 \rho}\left[\frac{1}{4} \bar{\alpha}_{s}\left(Q_{s}\right) b \ln ^{3}\left(Q_{s}^{2} r^{2}\right)+\left(1+\bar{\alpha}_{s}\left(Q_{s}\right)\left(\frac{67}{36}-\frac{\pi^{2}}{12}-\frac{5 N_{\mathrm{f}}}{18 N_{\mathrm{c}}}\right)\right) \ln ^{2}\left(Q_{s}^{2} r^{2}\right)-2 \zeta(3) \ln \left(Q_{s}^{2} r^{2}\right)\right]\right\} .
$$

We would like to point out that the two solutions, Eqs. (A5) and (A19), are consistent with each other, although the coefficients of the corresponding terms look different due to different handling methods of the running coupling and saturation momentum used in this paper and in Ref. [31].

[1] L. Gribov, E. Levin, and M. Ryskin, Phys. Rep. 100, 1 (1983).

[2] I. Balitsky, Nucl. Phys. B463, 99 (1996).

[3] Yu. V. Kovchegov, Phys. Rev. D 60, 034008 (1999).

[4] E. A. Kuraev, L. N. Lipatov, and V. S. Fadin, Sov. Phys. JETP 45, 199 (1977).

[5] I. Balitsky and L. N. Lipatov, Sov. J. Nucl. Phys. 28, 822 (1978).

[6] A. Mueller and D. Triantafyllopoulos, Nucl. Phys. B640, 331 (2002).

[7] E. Iancu, K. Itakura, and L. McLerran, Nucl. Phys. A708, 327 (2002).

[8] J. L. Albacete, N. Armesto, J. G. Milhano, C. A. Salgado, and U. A. Wiedemann, Phys. Rev. D 71, 014003 (2005).

[9] I. Balitsky, Phys. Rev. D 75, 014001 (2007).

[10] Yu. V. Kovchegov and H. Weigert, Nucl. Phys. A784, 188 (2007).

[11] W. Xiang, Phys. Rev. D 79, 014012 (2009).

[12] J. L. Albacete and Yu. V. Kovchegov, Phys. Rev. D 75, 125021 (2007).

[13] I. Balitsky and G. A. Chirilli, Phys. Rev. D 77, 014019 (2008).
[14] T. Lappi and H. Mäntysaari, Phys. Rev. D 91, 074016 (2015).

[15] E. Iancu, J. Madrigal, A. Mueller, G. Soyez, and D. Triantafyllopoulos, Phys. Lett. B 744, 293 (2015).

[16] A. Mueller and A. Shoshi, Nucl. Phys. B692, 175 (2004).

[17] A. Mueller and S. Munier, Phys. Lett. B 737, 303 (2014).

[18] T. Liou, A. Mueller, and S. Munier, Phys. Rev. D 95, 014001 (2017).

[19] L. Dominé, G. Giacalone, C. Lorcé, S. Munier, and S. Pekar, Phys. Rev. D 98, 114032 (2018).

[20] E. Iancu and A. Mueller, Nucl. Phys. A730, 494 (2004).

[21] W. Xiang, S. Cai, and D. Zhou, Phys. Rev. D 95, 116009 (2017).

[22] J. Jalilian-Marian, A. Kovner, A. Leonidov, and H. Weigert, Nucl. Phys. B504, 415 (1997).

[23] J. Jalilian-Marian, A. Kovner, A. Leonidov, and H. Weigert, Phys. Rev. D 59, 014014 (1998).

[24] E. Iancu, A. Leonidov, and L. D. McLerran, Nucl. Phys. A692, 583 (2001).

[25] E. Ferreiro, E. Iancu, A. Leonidov, and L. D. McLerran, Nucl. Phys. A703, 489 (2002).

[26] A. H. Mueller, arXiv:hep-ph/0111244. 
[27] E. Levin and K. Tuchin, Nucl. Phys. B573, 833 (2000); A691, 779 (2001).

[28] B. Saez and E. Levin, Nucl. Phys. A870, 83 (2011).

[29] D. Triantafyllopoulos, Nucl. Phys. B648, 293 (2003).

[30] A. Mueller, Nucl. Phys. B643, 501 (2002).

[31] C. Contreras, E. Levin, R. Meneses, and I. Potashnikova, Phys. Rev. D 94, 114028 (2016).
[32] E. Iancu and A. Mueller, Nucl. Phys. A730, 460 (2004).

[33] M. Braun, Phys. Lett. B 483, 115 (2000).

[34] M. Braun, Eur. Phys. J. C 16, 337 (2000).

[35] A. Kovner, E. Levin, and M. Lublinsky, J. High Energy Phys. 08 (2016) 031.

[36] M. Kozlov and E. Levin, Nucl. Phys. A739, 291 (2004).

[37] T. Lappi and H. Mantysaari, Phys. Rev. D 93, 094004 (2016). 